\title{
Log-Domain Implementation of Complex Dynamics Reaction-Diffusion Neural Networks
}

\author{
Teresa Serrano-Gotarredona, Associate Member, IEEE, and Bernabe Linares-Barranco, Member, IEEE
}

\begin{abstract}
In this paper, we have identified a second-order reaction-diffusion differential equation able to reproduce through parameter setting different complex spatio-temporal behaviors. We have designed a log-domain hardware that implements the spatially discretized version of the selected reaction-diffusion equation. The logarithmic compression of the state variables allows several decades of variation of these state variables within subthreshold operation of the MOS transistors. Furthermore, as all the equation parameters are implemented as currents, they can be adjusted several decades. As a demonstrator, we have designed a chip containing a linear array of ten second-order dynamics coupled cells. Using this hardware, we have experimentally reproduced two complex spatio-temporal phenomena: the propagation of travelling waves and of trigger waves, as well as isolated oscillatory cells.
\end{abstract}

Index Terms-Analog circuit design, analog very large scale integration (VLSI), current mode, logdomain, neural networks, nonlinear circuits, piecewise-linear circuit, subthreshold, weak inversion.

\section{INTRODUCTION}

$\mathbf{M}$ ANY pattern formation and wave propagation phenomena that appear in nature can be described by systems of coupled nonlinear differential equations, generally known as reaction-diffusion equations [1]. These wave propagation phenomena are exhibited by systems belonging to very different scientific disciplines. For example, in neurophysiology, the propagation of electrical impulses through the nervous system and the propagation of the cardiac movement through the cardiac muscle are classical examples of active wave propagation. It is known that anomalies causing disorganization of autowave structures result into patient's clinical syndromes [2], [3]. Some groups have also investigated the application of active mediums in image processing tasks [4]. However, the huge amount of calculations involved in the simulations of these spatio/temporal phenomena makes the process tedious and extremely time consuming. In such an scenario, the existence of a specialized parallel/processing hardware able to reproduce these behaviors at very high speed, would be a valuable tool to study and understand more deeply these phenomena. Recently, there have been several research groups realizing circuit implementations of reaction- diffusion equations [5]-[7].

Manuscript received September 15, 2002. This work was supported by ONR under Research Grant "Design of High Density and Neuromorphic CNN Universal Chips as Image Microprocessors" and by a Spanish Grant TIC19990446-C02-02.

The authors are with the Instituto de Microelectrónica de Sevilla, Centro Nacional de Microelectrónica, Sevilla 41012, Spain (e-mail: terese@imse.cnm.es).

Digital Object Identifier 10.1109/TNN.2003.816374
The equations describing active wave propagation have the general form

$$
\frac{\partial}{\partial t} \boldsymbol{u}=\boldsymbol{f}(\boldsymbol{u})+D \nabla^{2} \boldsymbol{u}
$$

where $\boldsymbol{u}(\boldsymbol{x}, t)$ is an $m$-dimensional vector which defines the system state at position $\boldsymbol{x}$ (where $\boldsymbol{x} \in \mathcal{R}^{n}$ ) at time $t$. $\boldsymbol{f}(\boldsymbol{u})$ is a set of $m$ nonlinear functions of the state variable vector $\boldsymbol{u}$. $D$ is an $m \times m$ matrix. The diagonal elements of $D, D_{i}$ are known as the diffusion coefficients of the $i$ th state variable. And $\nabla^{2}$ is the Laplacian operator, which defined in $\mathcal{R}^{n}$ is

$$
\nabla^{2} u_{l}=\frac{\partial^{2} u_{l}}{\partial x_{1}^{2}}+\frac{\partial^{2} u_{l}}{\partial x_{2}^{2}}+\cdots+\frac{\partial^{2} u_{l}}{\partial x_{n}^{2}}, \quad l=1,2, \ldots, m .
$$

Table I contains some well-known reaction-diffusion equations commonly used in the literature [8]-[13] to model complex spatio-temporal behaviors that appear in several disciplines. All the equations in Table I correspond to the general form of (1). These equations can generate patterns and waves including travelling waves, trigger waves, and spiral waves.

These phenomena have been also successfully reproduced in case where the continuum medium description is approximated by a discretely spaced one [14]-[16]. If we do this spatial discretization, in two dimensions $n=2$, (1) takes the form

$$
\begin{aligned}
& \frac{d}{d t} \boldsymbol{u}(i, j)=f(\boldsymbol{u}(i, j))+D[\boldsymbol{u}(i+1, j) \\
& \quad+\boldsymbol{u}(i, j+1)+\boldsymbol{u}(i-1, j)+\boldsymbol{u}(i, j-1)-4 \boldsymbol{u}(i, j)]
\end{aligned}
$$

where $\boldsymbol{u}(i, j)$ is the vector of state variables for the cell located at spatial position $(i, j)$.

Equation (3) can be interpreted as a regular array of cells. Each cell has complex dynamics described by $m$ state variables and is coupled to its neighbors through linear synapses.

Equations in Table I, show different levels of complexity, that is, number of state variables, number and type of nonlinearities and number of couplings between state variables of adjacent cells. We selected an equation that captures all the complex spatio-temporal behaviors: Turing pattern formation, traveling waves, trigger waves and spiral waves propagation, while at the same time maintains the lowest possible level of complexity [16].

The selected equation that fulfills the above requirements is

$$
\begin{aligned}
& \frac{d u}{d t}=-f(u)-g v+D_{u} \nabla^{2} u \\
& \frac{d v}{d t}=\varepsilon\left(u+\beta-\gamma v+D_{v} \nabla^{2} v\right)
\end{aligned}
$$


TABLE I

REACTION-DiFfusion Equations RePorted IN LITERATURE to DESCRIBE ACTIVE SySTEMS PERTAINING to SEVERAL SCIENTIFIC DisCIPLINES

\begin{tabular}{|c|c|c|}
\hline Discipline & Equation & \\
\hline Genetic & Fisher's Equation & $\frac{\partial u}{\partial t}=u(1-u)+\nabla^{2} u$ \\
\hline Biology & Meinhardt-Gierer & $\begin{array}{l}\frac{\partial u}{\partial t}=\alpha \frac{u^{2}}{v}-\beta v+D_{1} \nabla^{2} u \\
\frac{\partial v}{\partial t}=\alpha u^{2}-\gamma v+D_{2} \nabla^{2} v\end{array}$ \\
\hline Thermodynamics & Brusselator & $\begin{array}{c}\frac{\partial u}{\partial t}=a-(b+1) u+u^{2} v+D_{1} \nabla^{2} u \\
\frac{\partial v}{\partial t}=b u-u^{2} v+D_{2} \nabla^{2} v\end{array}$ \\
\hline \multirow[t]{2}{*}{ Neurophysiology } & FitzHugh-Nagumo & $\begin{array}{c}\frac{\partial u}{\partial t}=-\left(\frac{u^{3}}{3}-u\right)+D_{1} \nabla^{2} u \\
\frac{\partial v}{\partial t}=\varepsilon(u-b v)\end{array}$ \\
\hline & Hodgin-Huxley & $\begin{array}{c}\frac{\partial u}{\partial t}=-f(u)+D_{1} \nabla^{2} u \\
\frac{\partial v}{\partial t}=g_{v}(u)(1-v)-h_{v}(u) v \\
\frac{\partial q}{\partial t}=g_{q}(u)(1-q)-h_{q}(u) q \\
\frac{\partial p}{\partial t}=g_{p}(u)(1-p)-h_{p}(u) p\end{array}$ \\
\hline Chemistry & Oregonator & $\begin{array}{c}\varepsilon \frac{\partial u}{\partial t}=u+v-\alpha u^{2}+D_{1} \nabla^{2} u \\
\frac{\partial v}{\partial t}=-v+\beta q-u v+D_{2} \nabla^{2} v \\
\delta \frac{\partial}{\partial t} q=u-q+D_{3} \nabla^{2} q\end{array}$ \\
\hline
\end{tabular}

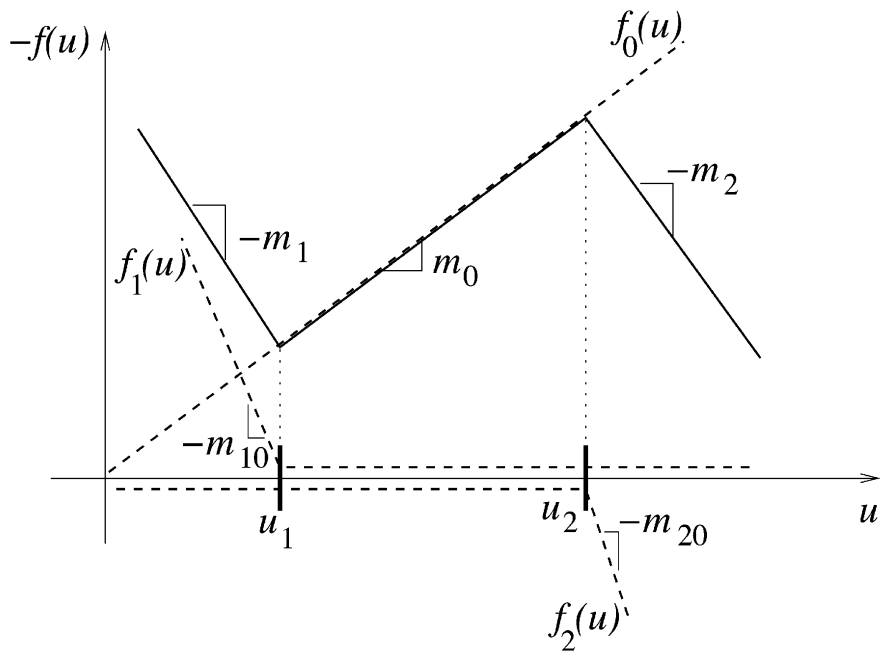

Fig. 1. Piecewise-linear approximation of the nonlinearity of (4).

where the nonlinear term $-f(u)$ has been approximated by the piecewise-linear nonlinearity depicted in Fig. 1.
Through proper parameter setting, this equation is able to exhibit Turing's patterns formation, trigger wave, travelling wave propagation and spiral wave formation. As will be demonstrated in the next Section, all the behaviors are robust against random variations in the equation parameters higher than $20 \%$ [16].

\section{BEHAVIORAL SimULATIONS}

The spatially discretized version of (4) have been simulated in MATLAB to reproduce the different spatio-temporal phenomena and to check the tolerance of these behaviors to random fluctuations in the parameters of (4).

A one-dimensional (1-D) array of $N=32$ cells has been simulated in MATLAB. The cell parameters were set to obtain two different behaviors: a travelling wave propagating with constant amplitude through the array and a trigger wave behavior. We have found points in the parameter space where the traveling wave behavior is robust against cell parameters random deviations as wide as $20 \%$. Deviations were introduced not only in the cell parameters, but also in the coupling parameters $D_{u}$ and $D_{v}$. 
TABLE II

TOLERANCE IN THE PARAMETER DEVIATIONS OF DifFERENT SPATIO-TEMPORAL BeHAVIORS

\begin{tabular}{c|c|c|c|c|c|c|c|c|c|c|c|c}
\hline & $m_{0}$ & $m_{1}$ & $m_{2}$ & $u_{1}$ & $u_{2}$ & $\varepsilon$ & $\beta$ & $\gamma$ & $g$ & $D_{u}$ & $D_{v}$ & $\sigma$ \\
\hline \hline travelling wave & -0.9 & 1 & 1 & -1 & 1 & $1 / 35$ & 0.55 & 1.2 & 1.1 & 0.5 & 0 & $25 \%$ \\
\hline trigger wave & -0.9 & 1 & 1 & -1 & 1 & $1 / 35$ & 0.7 & 5 & 1.2 & 0.5 & 0 & $30 \%$ \\
\hline spiral wave & -0.5 & 1 & 1 & -1 & 5 & $1 / 35$ & 0.7 & 1.2 & 1 & 0.1 & 0 & $20 \%$ \\
\hline $\begin{array}{c}\text { Turing's } \\
\text { patterns }\end{array}$ & -0.1 & 1 & 1 & -1 & 10 & $1 / 35$ & 0 & 6 & 1 & 0.1 & 50 & $30 \%$ \\
\hline
\end{tabular}
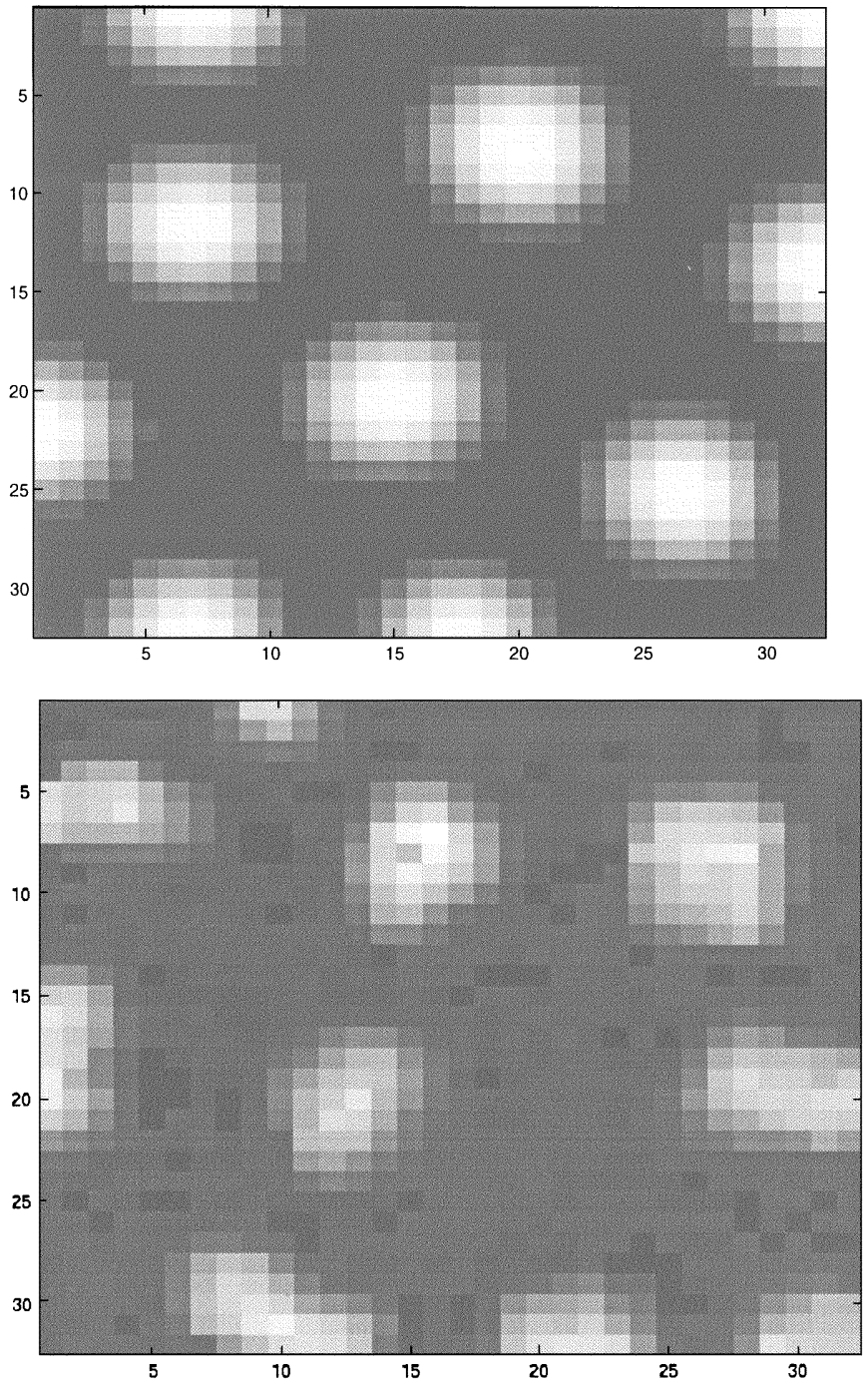

Fig. 2. Simulation of the formation of Turing's patterns in an array of $N \times$ $N=32 \times 32$ cells. Final pattern obtained in the $u$ state variable with the $u$ values coded in a scale of gray levels. (top) Simulation results when the cell parameters are set to their nominal values. (bottom) Simulation results when the cell parameters are generated with a deviation of $\sigma=30 \%$ around the nominal values.

The robustness of the behavior has also been checked for the trigger wave propagation. A tolerance of a standard deviation of $20 \%$ in the cell parameters and coupling parameters has been found through MATLAB simulations.

Simulations of the different behaviors in a two dimensional array of $N \times N=32 \times 32$ cells have also been performed.

Table II shows, for different spatio-temporal behaviors, the maximum standard deviations allowed simultaneously in all the cell parameters and coupling parameters that maintain the same qualitative behavior in all the cells in the array. Also shown in Table II, are the nominal cell and coupling parameters used in the different simulations.

The robustness of Turing's patterns formation against random parameter deviations is illustrated in Fig. 2. Fig. 2(top) depicts the Turing's patterns developed in the two-dimensional (2-D) array when the cell parameters are set to the values $m_{1}=m_{2}=$ $1, m_{0}=-0.1, u_{1}=-1, u_{2}=10, \varepsilon=1 / 35, \beta=0.0$, $\gamma=6, g=1$ and coupling parameters $D_{u}=0.1$ and $D_{v}=50$. Afterwards, a simulation with random parameters deviations of $\sigma=30 \%$ was performed. The deviations were introduced not only in the cell parameters but also in the coupling parameters between cells. Fig. 2(bottom) shows the result of this simulation. Fig. 2 shows the final patterns developed in the $u$ state variable with the values of the $u$ state variable coded as levels of gray.

As deduced from our system simulations, the transistors in our design must be dimensioned in order to ensure that the parameters in our hardware implementation have a standard deviation lower that $20 \%$. This level of precision is achievable in subthreshold MOS implementations for moderate transistor sizes. MOS transistors operating in subthreshold have a relative current precision almost independent of the operating current level [17]. Transistors of size $W=5 \mu \mathrm{m}, L=5 \mu \mathrm{m}$ fabricated in the AMS $0.35 \mu \mathrm{m}$ technology have in the weak inversion regime a standard deviation $\sigma(\Delta I) / I=2.7 \%$ [18]. In the rest of the paper, simulations of tolerance at the transistor level are omitted. The reason is that transient simulations of large arrays of cells have to be performed. These simulations at the transistor level would require days of computing.

\section{CIRCUIT SyNTHESIS}

Our target is to synthesize an array of second-order cells ( $m=2$ ) implementing the discretely spaced version of the nonlinear differential (4)

$$
\begin{aligned}
& \tau \frac{d}{d t} u(i, j) \\
= & -f(u(i, j))-g v(i, j)+D_{u}[u(i+1, j) \\
& +u(i, j+1)+u(i-1, j)+u(i, j-1)-4 u(i, j)] \\
& i=1,2, \ldots, N \\
& \frac{\tau}{\varepsilon} \frac{d}{d t} v(i, j) \\
= & u(i, j)+\beta-\gamma v(i, j)+D_{v}[v(i+1, j) \\
& +v(i, j+1)+v(i-1, j)+v(i, j-1)-4 v(i, j)] \\
& j=1,2, \ldots, N
\end{aligned}
$$

were the continuous 2-D Laplacian operator has been substituted by its discretized version [see (3)]. 
In order to reproduce different spatio-temporal phenomena through parameter setting, the hardware must have the following properties.

1) Parameters must be tunable in a wide range without degrading the behaviors.

2) State variables $u$ and $v$ must have a wide range of operation without saturation of the behavior.

3) For some locomotion applications, the time constant must be scalable to arbitrarily low values.

These requirements are met in a log-domain implementation [19]-[22]. In a log-domain circuit, the voltages in the circuit nodes are a logarithmically compressed version of the state variables. Thus, the state variables can vary several decades without saturation of the behavior. Also the cell parameters are currents that can be adjusted several decades in the MOS subthreshold regime [17], [23]. Furthermore, it is possible to scale down simultaneously all the currents in the circuit making the operation arbitrarily slow.

In the proposed circuit implementation we have to distinguish between mathematical state variables and circuital state variables. In the log-domain circuit technique, state variables $\left\{u_{n}(i, j), v_{n}(i, j)\right\}$ are currents and are related to node voltages $\left\{v_{u}(i, j), v_{v}(i, j)\right\}$ through exponential relationships

$$
\begin{aligned}
& u_{n}=I_{s} \exp \left(\alpha v_{u}\right) \\
& v_{n}=I_{s} \exp \left(\alpha v_{v}\right) .
\end{aligned}
$$

This means that the circuital state variables $\left\{u_{n}, v_{n}\right\}$ cannot be negative. Consequently, a coordinate transformation is required [21] to map the mathematical state variables $\{u, v\}$ in (5) to the circuital ones $\left\{u_{n}, v_{n}\right\}$

$$
\begin{aligned}
& u_{n}=\delta u+u_{0} \\
& v_{n}=\delta v+v_{0}
\end{aligned}
$$

where $\left(u_{0}, v_{0}\right)$ is the translation vector and $\delta$ is the scaling factor.

After substituting the original state variables, $u$ and $v$, of (4) by the scaled and translated variables $u_{n}$ and $v_{n}$, the following differential equation results:

$$
\begin{gathered}
\tau \frac{d u_{n}}{d t}=-f\left(u_{n}\right)-g v_{n}+u_{o f f}+D_{u} \nabla^{2} u_{n} \\
\frac{\tau}{\varepsilon} \frac{d v_{n}}{d t}=u_{n}+\beta_{n}-\gamma v_{n}+v_{o f f}+D_{v} \nabla^{2} v_{n}
\end{gathered}
$$

where $\beta_{n}=\delta \beta$, and the breakpoints of the piecewise-linear nonlinearity have been redefined as

$$
\begin{aligned}
& u_{1_{n}}=\delta u_{1}+u_{0} \\
& u_{2_{n}}=\delta u_{2}+u_{0} .
\end{aligned}
$$

Terms $u_{o f f}$ and $v_{o f f}$ are two offset terms defined as follows:

$$
\begin{aligned}
& u_{o f f}=g v_{0}+m_{0} u_{0} \\
& v_{o f f}=-u_{0}+\gamma v_{0} .
\end{aligned}
$$

Applying the log-domain mapping given by (6) to the discretely spaced version of (8) that results after the translation and scaling of the state variables, yields the following relation between circuit voltages:

$C_{u} \dot{v}_{u}=-f(u) e^{-\alpha v_{u}}-I_{g} e^{\alpha\left(v_{v}-v_{u}\right)}+I_{u o} e^{-\alpha v_{u}}+$ Coupling $_{u}$ $C_{v} \dot{v}_{v}=I_{e} e^{\alpha\left(v_{u}-v_{v}\right)}+I_{\beta} e^{-\alpha v_{v}}-I_{\gamma}+$ Coupling $_{v}$ where the terms called Coupling account for the Laplacian operator and are given as

$$
\begin{aligned}
\text { Coupling }_{u}= & I_{d u}\left(e^{\alpha\left(v_{u}(i+1, j)-v_{u}\right)}+e^{\alpha\left(v_{u}(i, j+1)-v_{u}\right)}\right. \\
& \left.+e^{\alpha\left(v_{u}(i-1, j)-v_{u}\right)}+e^{\alpha\left(v_{u}(i, j-1)-v_{u}\right)}-4\right) \\
\text { Coupling }_{v}= & I_{d v}\left(e^{\alpha\left(v_{v}(i+1, j)-v_{v}\right)}+e^{\alpha\left(v_{v}(i, j+1)-v_{v}\right)}\right. \\
& \left.+e^{\alpha\left(v_{v}(i-1, j)-v_{v}\right)}+e^{\alpha\left(v_{v}(i, j-1)-v_{v}\right)}-4\right) .
\end{aligned}
$$

Notice that the translated and scaled state variables have been renamed as $u$ and $v$ for shortness of notation. Parameters in (11) and (12) are related with the ones in (8) through the following relations:

$$
\begin{aligned}
C_{u} & =\alpha \tau I_{s} \\
\frac{C_{v}}{C_{u}} & =\frac{I_{e}}{\left(\varepsilon I_{s}\right)} \\
I_{g} & =g I_{s} \\
I_{u o} & =u_{o f f} \\
I_{d u} & =D_{u} I_{s} \\
I_{\beta} & =\left(\beta_{n}+v_{o f f}\right) \frac{I_{e}}{I_{s}} \\
I_{\gamma} & =\gamma I_{e} \\
I_{d v} & =D_{v} I_{e}
\end{aligned}
$$

where, as it will become clear later, in our implementation $I_{s}$ is a free design current parameter that for a given capacitance $C_{u}$ controls the characteristic time constant $\tau$ of the response. Reducing current $I_{s}$, the global response of the circuit can be appropriately slowed down. $I_{e}$ is also a current parameter that, for a given relation between capacitances $C_{v} / C_{u}$, must be tuned to set the original parameter $\varepsilon$ to its appropriate value.

Circuit implementation is focused on the realization of (11) and (12), and will be explained in two steps:

- implementation of the core cell dynamics (11), excluding coupling,

- implementation of the coupling terms (12).

\section{A. Uncoupled Cell}

Each uncoupled cell has a second-order dynamics described by

$$
\begin{aligned}
& C_{u} \dot{v}_{u}=-f(u) e^{-\alpha v_{u}}-I_{g} e^{\alpha\left(v_{v}-v_{u}\right)}+I_{u o} e^{-\alpha v_{u}} \\
& C_{v} \dot{v}_{v}=I_{e} e^{\alpha\left(v_{u}-v_{v}\right)}+I_{\beta} e^{-\alpha v_{v}}-I_{\gamma}
\end{aligned}
$$

as derived from (11) by making the coupling terms null.

Fig. 3 shows the block diagram of the circuit implementing (14). The blocks denoted as $E+$ and $E-$ are transconductors obeying an exponential law called $E$-elements [20], [21]. That is, the output current of these transconductors depends exponentially on the difference between the positive and negative controlling voltages. Fig. 4(a) shows the schematics of the bipolar implementation of the E-element. The circuit in Fig. 4(a) belongs to the class of translinear networks [24]. Using a reformulation of the translinear principle [25], this circuit can be easily 


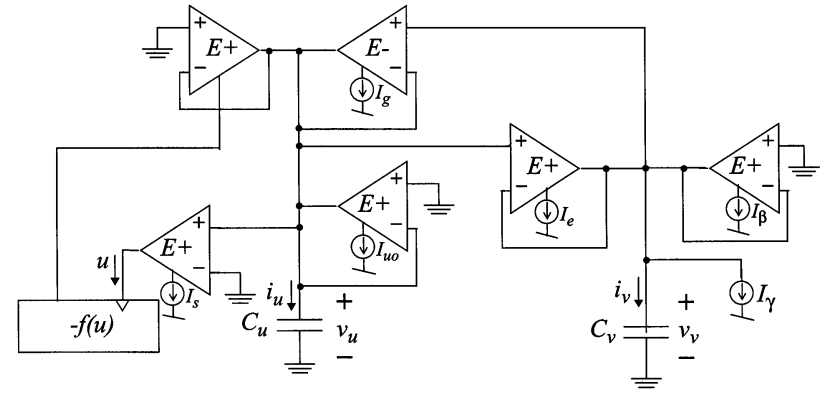

Fig. 3. Block diagram of the second-order circuit implementing (14).

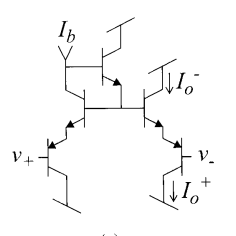

(a)



(b)

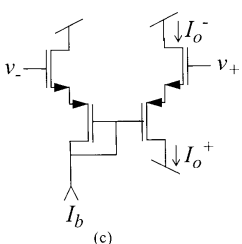

Fig. 4. E-element. (a) Bipolar implementation. (b) and (c) Two possible subthreshold MOS implementations.

analyzed. It is found that the circuit in Fig. 4(a) gives as output currents

$$
\begin{aligned}
& I_{o}^{+}=I_{b} \exp \left(\frac{v_{+}-v_{-}}{2 U_{T}}\right) \\
& I_{o}^{-}=-I_{b} \exp \left(\frac{v_{+}-v_{-}}{2 U_{T}}\right)
\end{aligned}
$$

where $U_{T}$ is the thermal voltage. The current is considered positive when it goes out of the $E$-element; the corresponding $E$-element is then considered a positive $E$-element. The current is negative when it enters the $E$-element, and the $E$-element is then called a negative $E$-element.

Fig. 4(b) and (c) shows two possible implementations of an E-element using MOS transistors operating in the subthreshold regime. The circuit in Fig. 4(b) verifies also an exact translinear relationship giving an output current

$$
I_{o}=I_{b} \exp \left(\frac{\kappa_{p}\left(v_{+}-v_{-}\right)}{2 U_{T}}\right)
$$

where $\kappa_{p}$ is a PMOS subthreshold parameter [17]. The output current of the circuit in Fig. 4(c) would be

$$
I_{o}=I_{b} \exp \left(\frac{\kappa_{n}\left(v_{+}-v_{-}\right)}{2 U_{T}}\right) .
$$

In our particular implementation we have chosen to implement all the $E$-elements using the block shown in Fig. 4(c). It is easy to show that with this selection of the transconductance elements, the circuit of Fig. 3 implements the system of differential equations given by (14) with

$$
\alpha=\frac{\kappa_{n}}{\left(2 U_{T}\right)} \text {. }
$$

The block denoted as $-f(u)$ in Fig. 3 implements in current mode the piecewise-linear nonlinearity shown in Fig. 1. A positive $E$-element biased by a current $I_{s}$ and controlled by the voltage difference $v_{+}-v_{-}=v_{u}$ (see Fig. 3 ) provides a sourcing current $u=I_{s} \exp \left(\alpha v_{u}\right)$. This current is used as the input of the nonlinear block $-f(u)$.

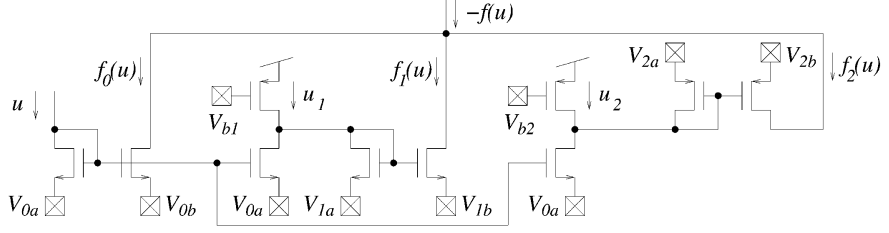

Fig. 5. Schematic of the piecewise-linear block.

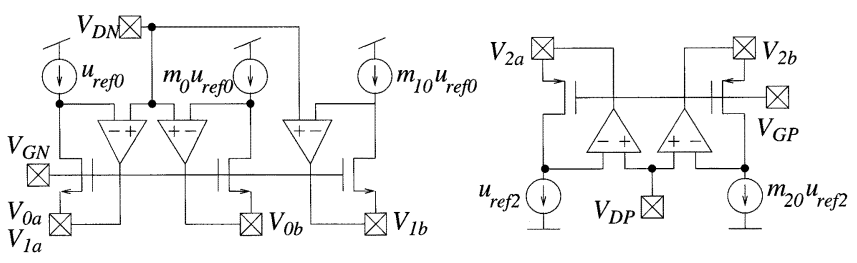

Fig. 6. Biasing of the piecewise-linear block.

The output current $-f(u)$ of this nonlinear block is the bias current of a positive $E$-element controlled by a voltage difference $v_{+}-v_{-}=-v_{u}$. Hence, a current term $(-f(u)) \exp \left(-\alpha v_{u}\right)$ is fed into the $C_{u}$ capacitor as required in (14).

\section{B. Piecewise-Linear Block}

As shown in Fig. 1, the PWL function $-f(u)$ can be decomposed as the sum of three components

$$
\begin{aligned}
-f(u) & =f_{0}+f_{1} u+f_{2}(u) \\
f_{0} u & =m_{0} u \\
f_{1}(u) & =\left(m_{1}+m_{0}\right)\left[u_{1}-u\right]^{+}=m_{10}\left[u_{1}-u\right]^{+} \\
f_{2}(u) & =-\left(m_{2}+m_{0}\right)\left[u-u_{2}\right]^{+}=-m_{20}\left[u-u_{2}\right]^{+}
\end{aligned}
$$

where $[x]^{+}$denotes the one-sided rectification operation $(x$ if $x \geq 0$ and zero if $x \leq 0), m_{0}, m_{1}$, and $m_{2}$ are the slopes of the three pieces of the nonlinearity and $u_{1}$ and $u_{2}$ are the breaking points.

Fig. 5 depicts the schematics of the current mode block that implements the PWL function of Fig. 1 [26]. The transistors are intended to operate in the subthreshold region, so that the slopes of the different pieces of the piecewise-linear characteristics can be controlled through the bias voltages $V_{0 a}, V_{0 b}, V_{1 a}, V_{1 b}, V_{2 a}$, and $V_{2 b}$. In particular

$$
\begin{aligned}
m_{0} & =\exp \left(\kappa_{n} \frac{V_{0 a}-V_{0 b}}{U_{T}}\right) \\
m_{10} & =\exp \left(\kappa_{n} \frac{V_{1 a}-V_{1 b}}{U_{T}}\right) \\
m_{20} & =\exp \left(\kappa_{p} \frac{V_{2 b}-V_{2 a}}{U_{T}}\right)
\end{aligned}
$$

However, the large variability of the factors $\kappa_{n}, \kappa_{p}$ and the current factors $I_{b}$ from run to run, makes impractical to control the slopes $m_{0}, m_{10}$, and $m_{20}$ by controlling directly the voltage biases. This inconvenience can be solved by using current biases to control the three slopes. Fig. 6 shows the biasing block which is common to all the cells in the chip. The amplifiers in Fig. 6 must deliver enough current for all the output branches in the different neurons. This means that the output stage of the amplifier must be scaled with the number of neurons in the chip. 


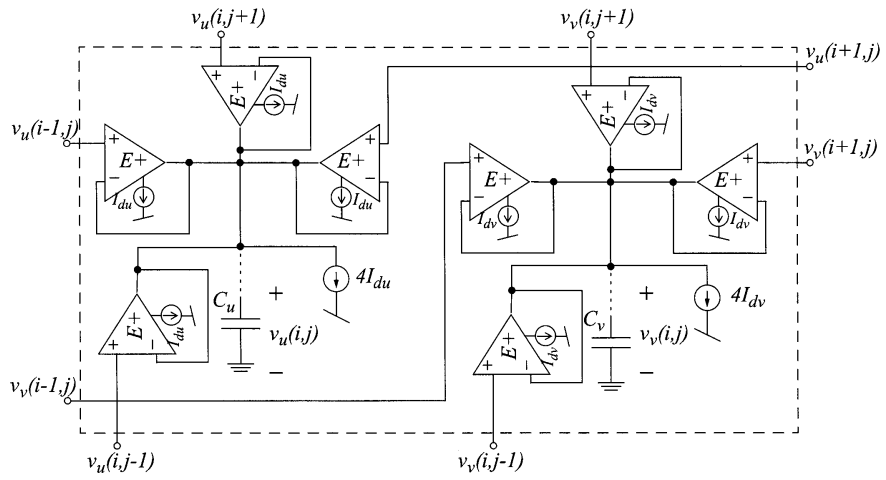

Fig. 7. Extra blocks needed to implement the coupling terms between cells.

\section{Coupling Between Cells}

To implement the coupling terms among cells in (12) eight additional $E$-elements are required per cell: four $E$-elements to couple the $v_{u}(i, j)$ voltage of cell $(i, j)$ with the $v_{u}$ voltages of its four nearest neighbors $v_{u}(i-1, j), v_{u}(i+1, j), v_{u}(i, j-1)$, and $v_{u}(i, j+1)$. Four other $E$-elements to couple the $v_{v}(i, j)$ voltage of each cell $(i, j)$ with the $v_{v}$ voltage of its four nearest neighbors $v_{v}(i-1, j), v_{v}(i+1, j), v_{v}(i, j-1)$, and $v_{v}(i, j+1)$. Fig. 7 shows a diagram of the extra blocks needed to implement the coupling terms that appear in (12).

\section{Initial Conditions Circuitry}

To generate the different spatio-temporal behaviors, appropriate initial conditions must be set in the $u$ and $v$ state variables of all the cells in the array [16]. In our design, binary initial conditions are used. That is, for each cell in the array, we set the initial values of the $u$ and $v$ state variables to one of two possible continuously programmable states $\left(u_{0_{1}}, v_{0_{1}}\right)$ or $\left(u_{0_{2}}, v_{0_{2}}\right)$. Afterwards, the system switches to its normal evolution mode, and different spatio-temporal behaviors are generated on chip, depending on the parameters and initial condition settings.

A careful design of the initial conditions circuitry is needed in order to avoid erasing or modification of the stored initial states during this switching. This switching problem is particularly severe in log-domain operation where the $u$ and $v$ state variables depend exponentially on the log-compressed $v_{u}$ and $v_{v}$ voltages stored on capacitors $C_{u}$ and $C_{v}$. Small deviations of $v_{u_{0}}$ or $v_{v_{0}}$ due to charge injection during the switching are exponentially amplified producing large errors in the initial state. Hence, avoiding any feedthrough injection during switching is essential for correct operation.

Fig. 8(a) depicts a block diagram of the circuitry added to set the initial conditions in the $v_{u}$ and $v_{v}$ voltages. A set of inverters configured as a shift register selects which initial conditions $\left(u_{0_{1}}, v_{0_{1}}\right)$ or $\left(u_{0_{2}}, v_{0_{2}}\right)$ are set in each cell. Fig. 8(b) shows the schematic of the boxed block in Fig. 8(a) that sets the initial voltage $v_{0_{i}}$ (or $u_{0_{i}}$ ) in cell capacitor $C_{u}$ (or $C_{v}$ ). In Fig. 8(b), $v_{s b}$ and $v_{s c}$ are two switched voltages. $v_{s c}$ switches from $V_{d d}$, when the initiation signal $i c$ is high, to $V_{d d}-1 \mathrm{~V}$, when signal $i c$ is low (normal operation mode). Signal $v_{s b}$ switches from $V_{s s}$, when $i c$ is high, to $V_{s s}+1 \mathrm{~V}$, when $i c$ is low. When signal $i c$ is high the initial conditions are being set in the cells. During this period, all the components in the circuit are disconnected from nodes $v_{u}$ (or $v_{v}$ ), except one of the boxed elements of Fig. 8(b).
In the steady state, when the input current to capacitor $C_{u}$ (or $C_{v}$ ) is zero, the output current of the negative $E$-element [current $i_{o e}$ in Fig. 8(b)], is forced to be equal to the supplied current $u_{0_{i}}$ (or $v_{0_{i}}$ ). Hence, the output voltage $v_{o}$ (which is connected to capacitor nodes $v_{u}$ or $v_{v}$ ) is forced to be

$$
v_{u}=\frac{1}{\alpha} \ln \left(\frac{u_{0_{i}}}{I_{s}}\right)
$$

hence

$$
u_{0_{i}}=I_{s} \exp \left(\alpha v_{u}\right)
$$

When signal $i c$ is low, currents $u_{0_{i}}$ (or $v_{0_{i}}$ ) and $i_{o e}$ are cut off. The switching of the currents is done by switching the source terminals of the current supplying transistors, with their gate terminal being kept at constant voltages. This switching strategy avoids any undesired charge injection in the output node [27].

As explained before, during the setting of the initial conditions all the other components in the circuit must be delivering a zero output current to the $v_{u}$ and $v_{v}$ nodes. This can be achieved by substituting all the $E$-elements and current sources in Figs. 3 and 7 by switched $E$-elements and switched current sources. The schematic of a switched positive $E$-element is shown in Fig. 9(a). Fig. 9(b) shows the schematic of the switched negative E-element. And Fig. 9(c) depicts the schematic of a switched sinking current source. Note that all the switching is done through the sources of the output current supplying transistors to avoid any charge injection in the capacitors during switching [27].

\section{EXPERIMENTAL RESULTS}

A 1-D array of $N=10$ coupled cells has been integrated in a $0.35-\mu \mathrm{m}$ three-metal double-poly CMOS technology provided by the AMS foundry. Each cell occupies an area of $116 \mu \mathrm{m}$ $\times 227 \mu \mathrm{m}$. Thus, an array of $32 \times 32$ cells would occupy in this technology an area of $27 \mathrm{~mm}^{2}$. Fig. 10 shows the layout of one cell in the array. About one third of the cell area corresponds to the cell capacitors $C_{u}=0.45 \mathrm{pF}$ and $C_{v}=4.5 \mathrm{pF}$, which are implemented as standard double-poly capacitors available in the technology. Each unit capacitor $C_{u}=0.45 \mathrm{pF}$ occupies an area of approximately $62 \mu \mathrm{m} \times 11.5 \mu \mathrm{m}$. The area of each $E$-element is approximately $50 \mu \mathrm{m} \times 15 \mu \mathrm{m}$.

Fig. 11 shows a microphotograph of the fabricated chip. In the prototype chip, we have also included an isolated current-mode piecewise-linear block $-f(u)$ and an isolated cell for characterization purposes.

\section{A. Current-Mode Piecewise-Linear Block}

All the biasing currents $u_{1}, u_{2}, u_{r e f 0}, m_{0} u_{r e f 0}, m_{10} u_{r e f 0}$, $u_{\text {ref } 2}, m_{20} u_{\text {ref } 2}$ (see Figs. 5 and 6) are derived from the same reference current $I_{\mathrm{REF}}$, through 5-bit DAC converters. The value of each current can be varied between $\left\{0, I_{\mathrm{REF}} / 16, \ldots, 2 I_{\mathrm{REF}}\right\}$. The functionality of the block has been verified for at least four decades of variation of the working currents. Fig. 12(a) shows the measured input-output current characteristics of the block for $u_{1}=(10 / 16) I_{\mathrm{REF}}, u_{2}=$ $(25 / 16) I_{\mathrm{REF}}, u_{\mathrm{ref0}}=(10 / 16) I_{\mathrm{REF}}, u_{\mathrm{ref} 2}=(10 / 16) I_{\mathrm{REF}}$, $m_{0} u_{\mathrm{ref} 0}=(10 / 16) I_{\mathrm{REF}}, m_{10} u_{\mathrm{ref0}}=(20 / 16) I_{\mathrm{REF}}$, $m_{20} u_{\mathrm{ref} 2}=(20 / 16) I_{\mathrm{REF}}$, and $I_{\mathrm{REF}}=50$ pA. Fig. 12(b) 

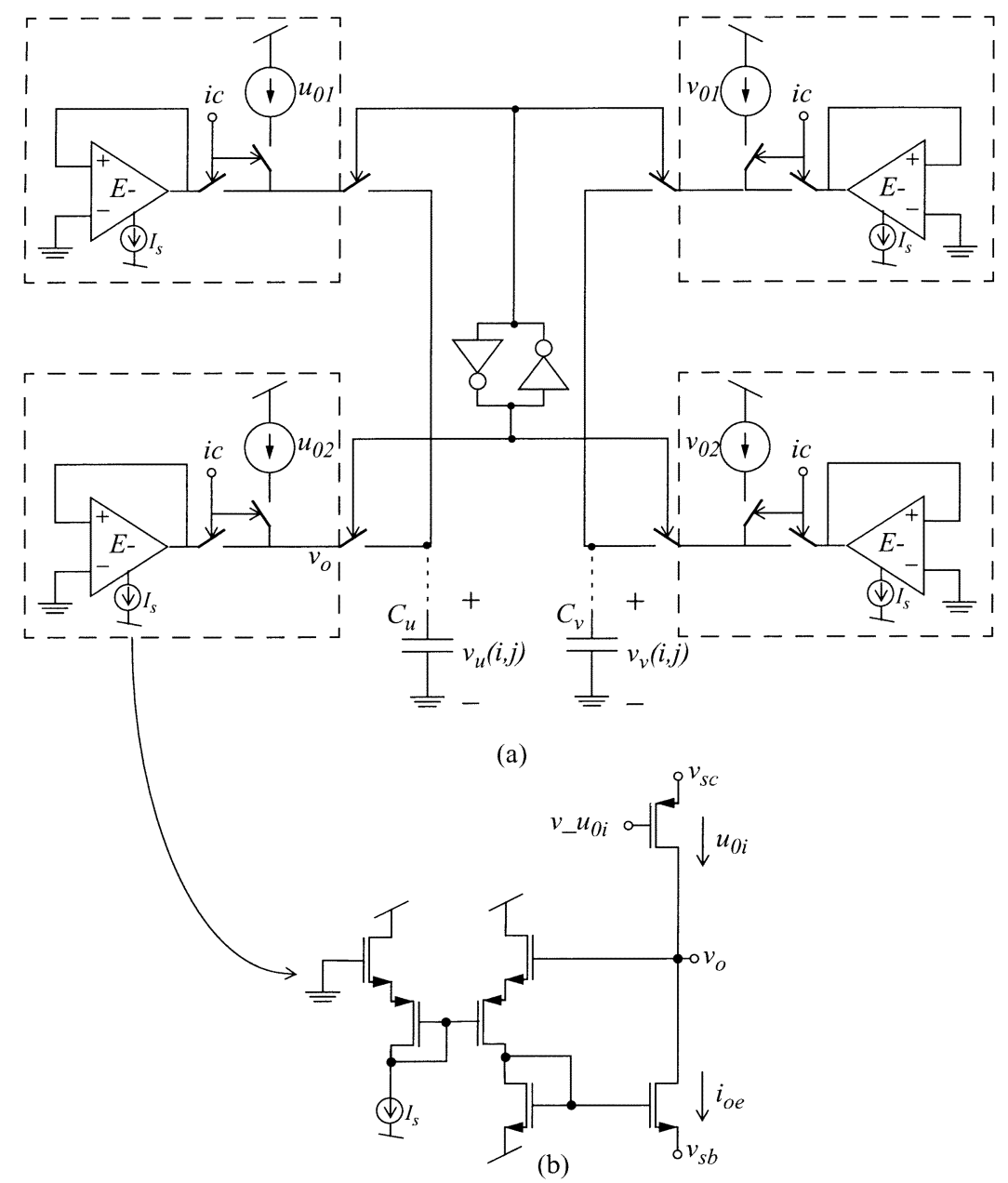

Fig. 8. (a) Block diagram of the initial conditions circuitry. (b) Schematic of the boxed block in Fig. 8(a).

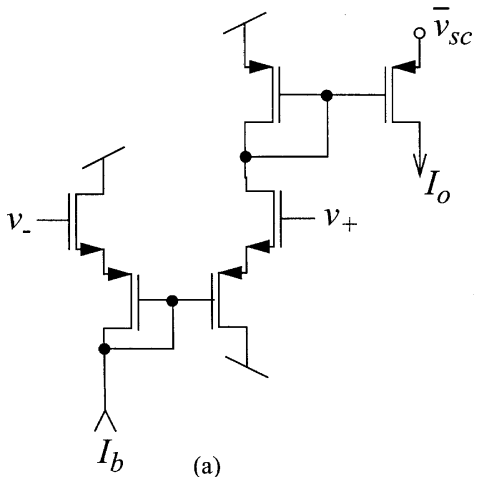

(a)

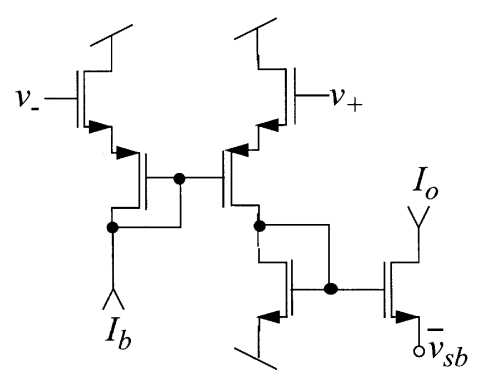

(b)

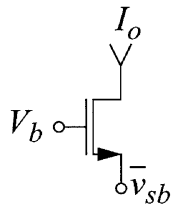

(c)

Fig. 9. Schematics of (a) switched E+-element, (b) switched $E$--element, and (c) switched current source.

depicts the measured input-output current characteristics for the same parameters but with $I_{\mathrm{REF}}=500 \mathrm{nA}$.

Fig. 13(a) illustrates the controllability of the central slope $m_{0}$. In the measurements of Fig. 13(a) $I_{\mathrm{REF}}=5 \mathrm{nA}$, $u_{1}=(10 / 16) I_{\mathrm{REF}}, u_{2}=(25 / 16) I_{R E F}, u_{\mathrm{ref} 0}=(10 / 16) I_{\mathrm{REF}}$, $u_{\mathrm{ref} 2}=(10 / 16) I_{\mathrm{REF}}$, while $m_{0} u_{\mathrm{ref0}}$ is varied from 0 to $(10 / 16) I_{\mathrm{REF}}$. The values of $m_{10} u_{\mathrm{ref0}}$ and $m_{20} u_{\mathrm{ref} 2}$ are simultaneously swept from $(10 / 16) I_{\mathrm{REF}}$ to $(20 / 16) I_{\mathrm{REF}}$, so that as can be observed the $m_{1}$ and $m_{2}$ slopes remain constant and approximately equal to one. Fig. 13(b) illustrates the programma- bility of the $m_{2}$ slope. In these curves, $u_{1}=(10 / 16) I_{\mathrm{REF}}, u_{2}=$ $(25 / 16) I_{\mathrm{REF}}, u_{\mathrm{ref} 0}=(10 / 16) I_{\mathrm{REF}}, u_{\mathrm{ref} 2}=(10 / 16) I_{\mathrm{REF}}$, $m_{0} u_{\mathrm{ref} 0}=(10 / 16) I_{\mathrm{REF}}, m_{10} u_{\mathrm{ref} 0}=(20 / 16) I_{\mathrm{REF}}$ and $I_{\mathrm{REF}}=5 \mathrm{nA}$, while $m_{20} u_{\mathrm{ref} 2}$ is varied from 0 to $2 I_{\mathrm{REF}}$. The controllability of the breaking point $u_{2}$ is demonstrated in the measurements of Fig. 13(c). In the curves of Fig. 13(c), $u_{1}=(10 / 16) I_{\mathrm{REF}}, u_{\mathrm{refO}}=(10 / 16) I_{\mathrm{REF}}$, $u_{\mathrm{ref} 2}=(10 / 16) I_{\mathrm{REF}}, \quad m_{0} u_{\mathrm{ref} 0}=(10 / 16) I_{\mathrm{REF}}$, $m_{10} u_{\mathrm{ref} 0}=(20 / 16) I_{\mathrm{REF}}, m_{20} u_{\mathrm{ref} 2}=(20 / 16) I_{\mathrm{REF}}$, $I_{\mathrm{REF}}=5 \mathrm{nA}$, and $u_{2}$ varies from $(11 / 16) I_{\mathrm{REF}}$ to $2 I_{\mathrm{REF}}$. 


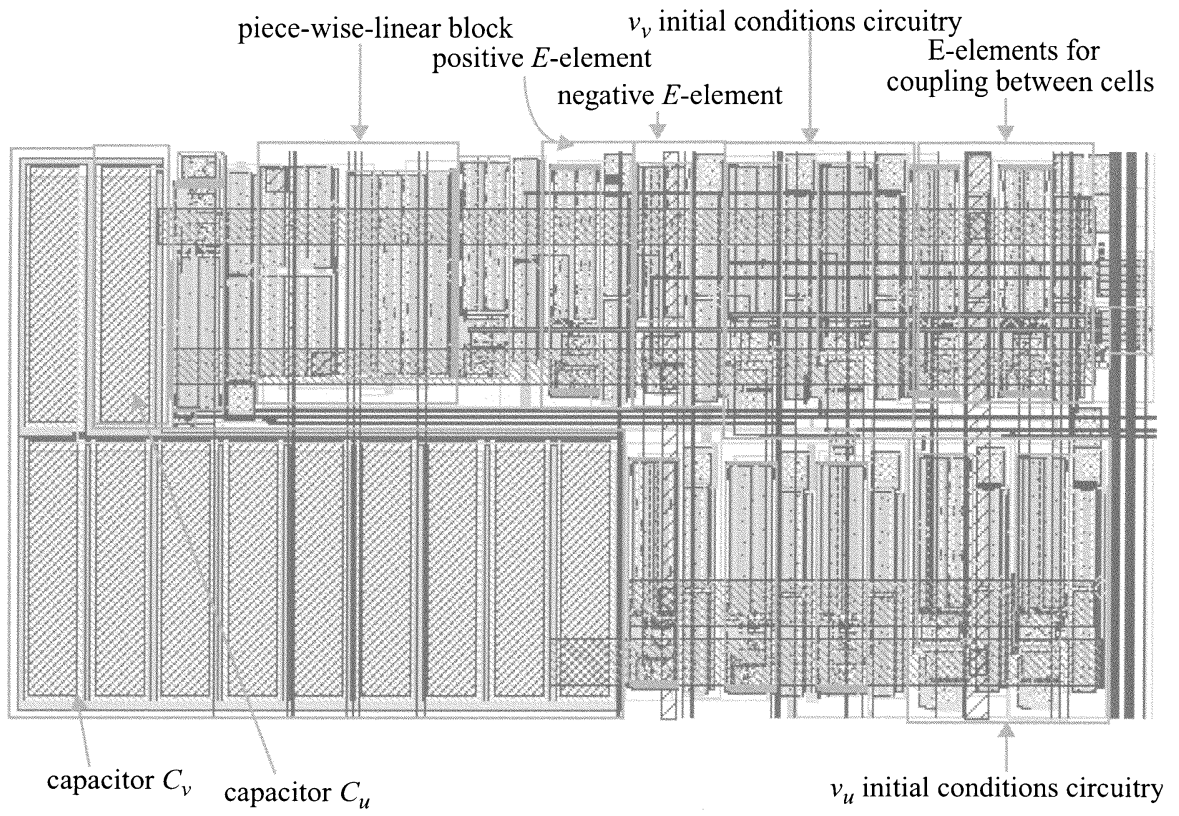

Fig. 10. Layout of one cell.

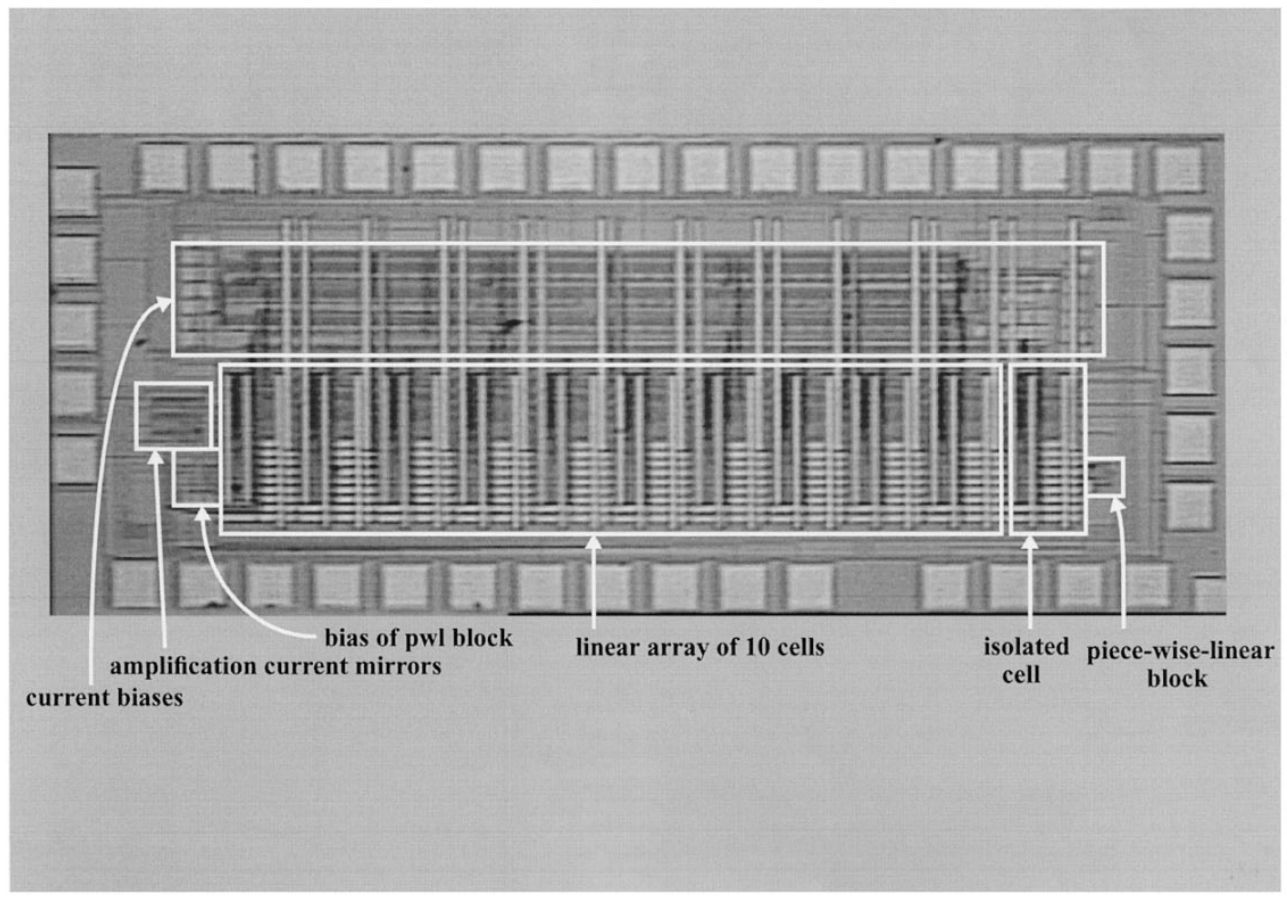

Fig. 11. Microphotograph of the fabricated chip.

\section{B. Isolated Cell}

An isolated cell where the nodes $v_{u}$ and $v_{v}$ (see Fig. 3) are directly connected to external pins has been included in the prototype chip. This allows us to do a complete dc characterization of the different sub blocks in the cell.

Fig. 14 shows measurements of the input-output characteristics of a positive $E$-element. The measured $E$-element is the one whose bias current is $I_{e}$. In these measurements, bias currents $I_{\beta}$ and $I_{\gamma}$ were set to zero. Fig. 14(top) shows current $i_{v}$ versus the voltage applied to this node $v_{v}$, for different values of the voltage applied to node $v_{u}$. Voltage $v_{u}$ was swept from 1.2 to $1.8 \mathrm{~V}$ in $50-\mathrm{mV}$ steps. The bias current $I_{e}$ was held constant at $1 \mathrm{nA}$. Fig. 14(bottom) depicts current $i_{v}$ versus the voltage applied to this node $v_{v}$, for different values of the bias current $I_{e}$. In these 32 curves, the bias current $I_{e}$ was varied linearly in the range $\left[1 \mathrm{nA} / 16,2 \mathrm{nA}\right.$ ], while voltage $v_{u}$ was held constant at $1.5 \mathrm{~V}$. The dots in Fig. 14 correspond to the experimentally measured points. The solid lines are the theoretical curves

$$
I_{o}=I_{e} \exp \left(\kappa_{n} \frac{v_{u}-v_{v}}{2 U_{T}}\right)
$$

fitted for a value $\kappa_{n}=0.81$. 


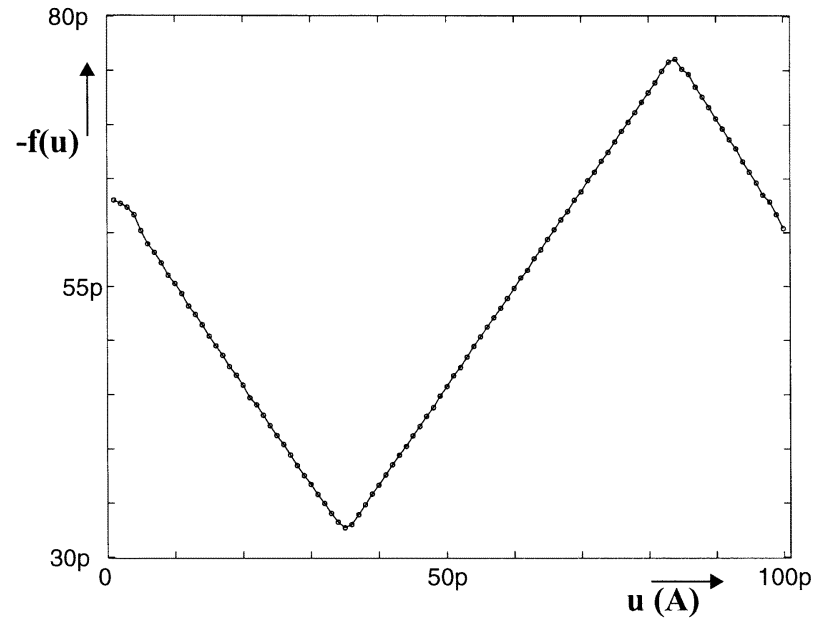

(a)

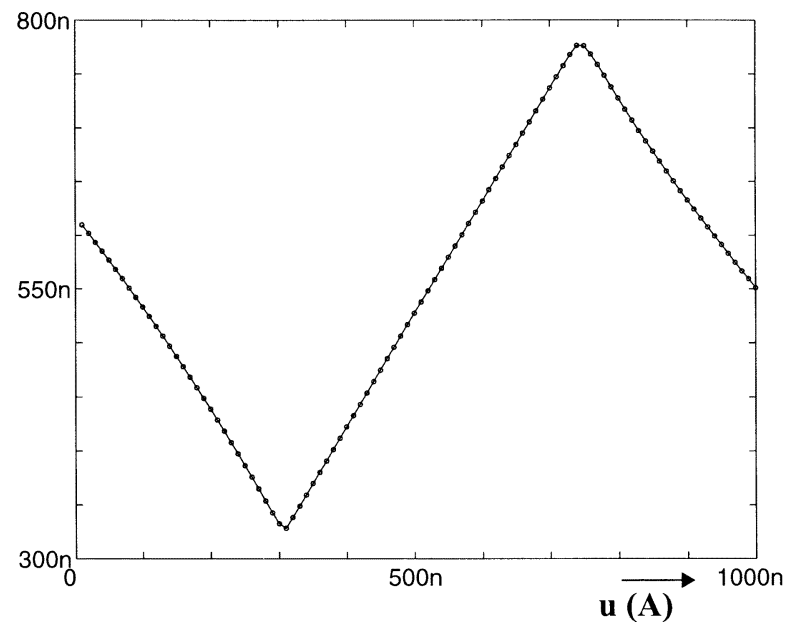

(b)

Fig. 12. Input-output behavior of the piecewise linear block for (a) input current $u \in[0,100 \mathrm{pA}]$ and (b) $u \in[0,1 \mu \mathrm{A}]$.

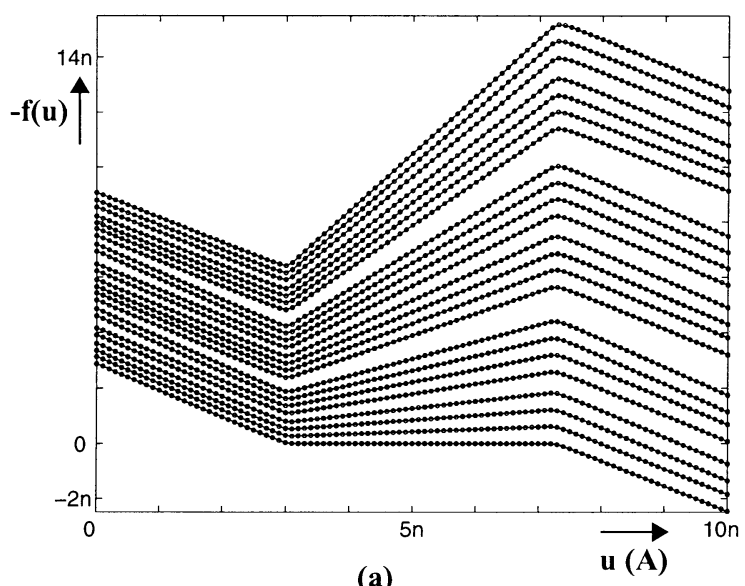

(a)

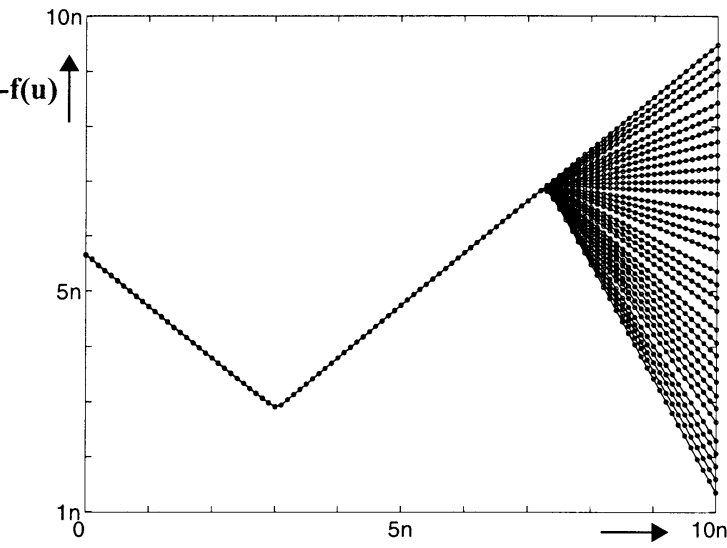

(b)

u (A)

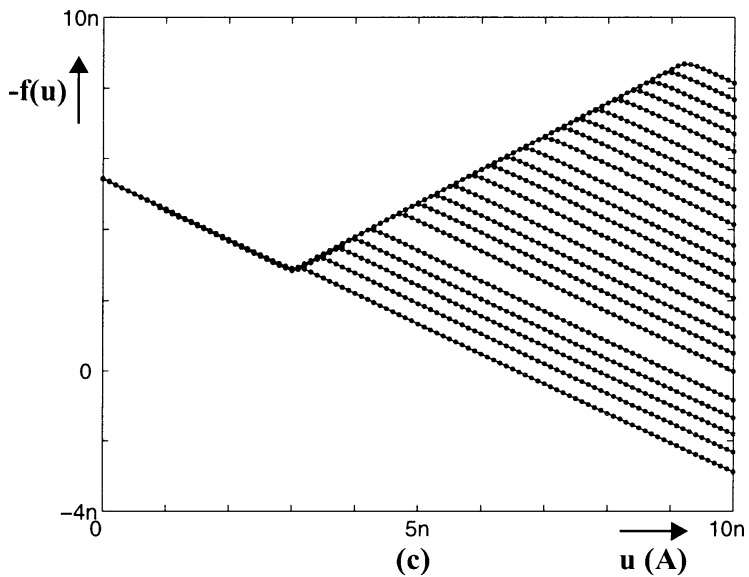

Fig. 13. Measured input-output characteristic. (a) For different values of the central slope $m_{0}$. (b) For different values of the $m_{2}$ slope. (c) For different values of the $u_{2}$ breaking point.

Fig. 15 shows measured results of a negative $E$-element. The measured negative E-element is the one with bias current $I_{g}$ in Fig. 3. Bias currents $I_{u o}$ and $I_{s}$ were set to zero is these measurements. Fig. 15(top) shows current $i_{u}$ versus the applied voltage $v_{u}$ for different values of the voltage applied to node $v_{v}$ with current $I_{g}$ at $1 \mathrm{nA}$. Fig. 15(bottom) plots current $i_{u}$ versus the voltage applied to this node $v_{u}$, for different values of the bias current $I_{g}$. In these 32 curves, the bias current $I_{g}$ was varied linearly in the range [1 nA/16, $2 n A]$, while voltage $v_{v}$ was held constant at $1.5 \mathrm{~V}$. The dots in Fig. 15 correspond to the experimentally measured points. The solid lines are the theoretically predicted curves

$$
I_{o}=I_{g} \exp \left(\kappa_{n} \frac{v_{v}-v_{u}}{2 U_{T}}\right)
$$

fitted for the same value $\kappa_{n}=0.81$. 



Fig. 14. DC characteristics of a positive $E$-element: Output current versus voltage in the negative input (top) for different voltages applied in the positive input and (bottom) for different biasing currents.

We have also measured the null isoclines of the isolated cell for different settings of the parameters. To obtain the null isoclines, the following procedure has been followed.

1) For the null isoclines corresponding to $\dot{v}=0$, we sweep the $v_{u}$ voltage (or equivalently the $u=I_{s} \exp \left(\alpha v_{u}\right)$ state variable) and measure the voltage that appears in $\mathrm{dc}$ in the $v_{v}$ node (or equivalently the $v=I_{s} \exp \left(\alpha v_{u}\right)$ state variable) when the current $i_{v}$ flowing through that node is zero. The measured pairs $(u, v)$ correspond to the points that belong to the isocline $\dot{v}=0$.

2) For the null isoclines corresponding to $\dot{u}=0$, a two-dimensional sweep of voltages $v_{u}$ and $v_{v}$ is done. The total current $i_{u}$ flowing through node $v_{u}$ is measured and its zero crossings are observed. For each value of $v_{v}$ (or equivalently the $v=I_{s} \exp \left(\alpha v_{v}\right)$ state variable), we look for the corresponding values of $v_{u}$ (or equivalently the $u=I_{s} \exp \left(\alpha v_{u}\right)$ state variable) such that current $i_{u}$ becomes zero. The measured pairs $(u, v)$ correspond to the points that belong to the isocline curve $\dot{u}=0$.

Fig. 16 shows the $\dot{v}$ null isoclines measured for different settings of the circuit parameters. They are compared versus the ones theoretically computed using (8) for the equivalent equation parameters. The equivalent parameters are computed using the relations given by (13). Fig. 16(a) shows the $\dot{v}=0$ null isoclines measured for the circuit parameters: $I_{s}=1 \mathrm{nA}, I_{e}=$ 



Fig. 15. DC characteristics of a negative $E$-element: Output current versus voltage in the negative input (top) for different voltages applied in the positive input and (bottom) for different biasing currents.

$(5 / 16) 1 \mathrm{nA}, I_{\gamma}=(3 / 16) 1 \mathrm{nA}$, while $I_{\beta}$ was varied from $0 \mathrm{nA}$ to $2 \times 45 \mathrm{nA}$ in steps of $45 \mathrm{nA} / 16$. Also shown in Fig. 16(a) are the $\dot{v}$ null isoclines computed using (8) for $\gamma=3 / 5$ and varying $\beta$ from 0 to $32 \times 9 \mathrm{nA}$ in steps of $9 \mathrm{nA}$. Fig. 16(b) shows the $\dot{v}=0$ null isoclines measured for the circuit parameters: $I_{s}=1 \mathrm{nA}$, $I_{e}=(5 / 16) 1 \mathrm{nA}, I_{\beta}=1.4 \mathrm{nA}$, while $I_{\gamma}$ was varied from $1 / 16$ $\mathrm{nA}$ to $2 \mathrm{nA}$ in steps of $1 \mathrm{nA} / 16$. Also shown in Fig. 16(b) are the $\dot{v}$ null isoclines computed using (8) for $\beta=4.5 \mathrm{nA}$ and varying $\gamma$ from 0.2 to 6.4 in steps of 0.2 . A valid agreement is observed between the measured null isoclines and the theoretically computed ones.

Fig. 17 shows a comparison between the $\dot{u}=0$ null isoclines measured experimentally for different values of the circuit parameters and the ones theoretically computed using (8). In the measurements of Fig. 17(a), $I_{s}=1 \mathrm{nA}, I_{g}=1 \mathrm{nA}$, $I_{u o}=0 \mathrm{nA}, u_{1}=28.125 \mathrm{nA}, u_{2}=90 \mathrm{nA}$, and $m_{0}=1$. The dotted line was measured for $m_{10}=m_{20}=3.2$, while the line marked with asterixs corresponds to the points measured for $m_{10}=m_{20}=1.9$. The solid lines are the theoretically computed $\dot{u}=0$ null isoclines using ( 8 ) for $g=1, u_{\text {off }}=0$ and for the $-f(u)$ block parameters: $u_{1}=28.125 \mathrm{nA}, u_{2}=90 \mathrm{nA}$, $m_{0}=1$, and $m_{1}=m_{2}=2.2$, and $m_{1}=m_{2}=0.9$. Fig. 17(b) shows the $\dot{u}=0$ null isoclines for different values of the $g$ parameter. In the measurements depicted in Fig. 17(b) the circuit parameters are set to $I_{s}=1 \mathrm{nA}, I_{u o}=0 \mathrm{nA}, u_{1}=28.125 \mathrm{nA}$, $u_{2}=90 \mathrm{nA}, m_{0}=1, m_{10}=m_{20}=3.2$, and $I_{g}$ is set 



Fig. 16. Comparison between the experimentally measured $\dot{v}=0$ null isoclines and the ones computed using (8).

to $1 \mathrm{nA}$ (dotted line), $2 \mathrm{nA}$ (curve marked with diamonds) and $0.125 \mathrm{nA}$ (curve marked with asterixs). The theoretically computed curves using (8) and the equivalent parameters given by (13), are also shown in Fig. 17 with solid lines.

Using the measured $\dot{u}=0, \dot{v}=0$ null isoclines we can figure out how to set the cell parameters to obtain different qualitative behaviors. In the measurements of Fig. 18(a) the cell parameters are set to emulate an excitable medium [28]. That is, each cell has a unique stable equilibrium point. Under perturbation, the cells become excited and go back to their stable equilibrium point after a refractory period. The circuit parameters in Fig. 18(a) are: $I_{s}=1 \mathrm{nA}, I_{e}=0.3125 \mathrm{nA}$,
$I_{\beta}=5.625 \mathrm{nA}, I_{\gamma}=0.375 \mathrm{nA}, I_{g}=0.5625 \mathrm{nA}, I_{u o}=0 \mathrm{nA}$, and for the nonlinear block parameters, $m_{10}=3.2, m_{20}=3.2$, $m_{0}=1, u_{1}=28.125 \mathrm{nA}, u_{2}=90 \mathrm{nA}$.

The measurements of Fig. 18(b) correspond to the null isoclines $\dot{u}=0, \dot{v}=0$ of a bistable medium [28]. That is, each uncoupled cell has two stable equilibrium points. Under perturbation the cells can trigger from one equilibrium point to the other. The circuit parameters were set to: $I_{s}=1 \mathrm{nA}$, $I_{e}=0.3125 \mathrm{nA}, I_{\beta}=16.875 \mathrm{nA}, I_{\gamma}=0.375 \mathrm{nA}, I_{g}=1 \mathrm{nA}$, $I_{u o}=0$, and the same parameters the parameters are the same used in Fig. 18(a), that is, $m_{10}=3.2, m_{20}=3.2, m_{0}=1$, $u_{1}=28.125 \mathrm{nA}, u_{2}=90 \mathrm{nA}$. 

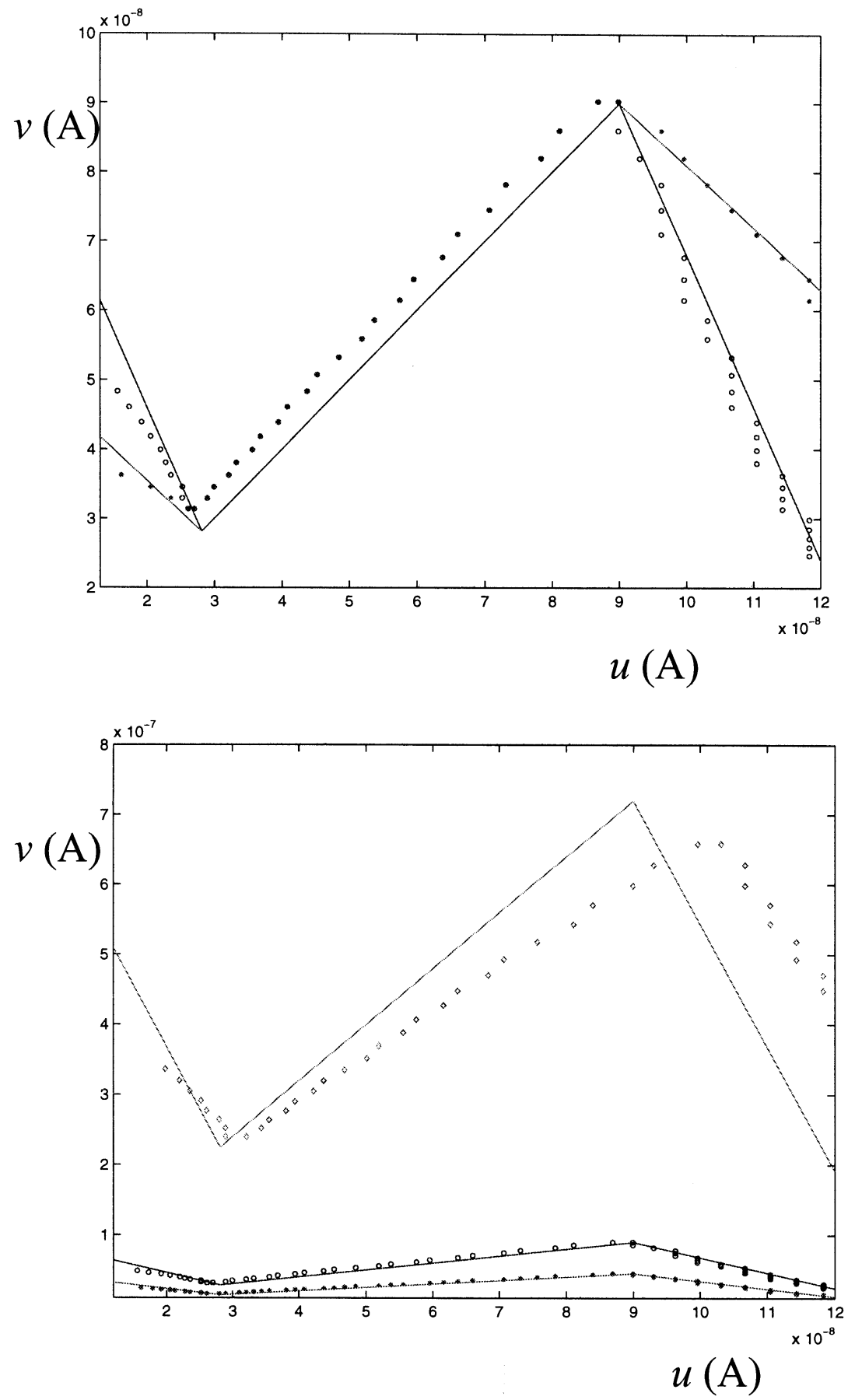

Fig. 17. Comparison between the experimentally measured $\dot{u}=0$ null isoclines and the ones computed using (8).

In the measurements shown in Fig. 18(c) the cell parameters are set such that the cells have a unique equilibrium point which is unstable. It is therefore an oscillatory medium. The cell parameters were set in this case to: $I_{s}=1 \mathrm{nA}, I_{e}=0.3125 \mathrm{nA}$, $I_{\beta}=0 \mathrm{nA}, I_{\gamma}=0.1875 \mathrm{nA}, I_{g}=0.5 \mathrm{nA}, I_{u o}=0.0625 \mathrm{nA}$, and for the nonlinear block parameters $m_{10}=1.9, m_{20}=1.9$, $m_{0}=1, u_{1}=0.625 \mathrm{nA}$, and $u_{2}=2 \mathrm{nA}$.

\section{1-D Array of Cells}

We have measured the transient behavior of a linear array of ten coupled cells. Each cell is coupled to its two nearest neighbors with the coupling strategy shown in Fig. 7. As shown in
Fig. 19, to observe the state variables $u, v$ of each cell two additional positive $E$-elements have been added per cell. They transform the circuit voltages $v_{u}, v_{v}$ to the equation state variables (currents $u, v$ ). The cell to be observed is selected through signal $s c_{i}$ and connected to a buffering amplification current mirror. This mirror is an NMOS active input current mirror [29]. The low input impedance is intended to minimize the delay in the input node. The mirror has a current amplification factor around 1000. This way, the low currents in the cells (in the order of nano Amperes) are transformed into higher currents (in the order of micro Amperes) to be sensed and transformed into voltages by off-chip resistors. The off-chip resistors used were $R_{u}=10 \mathrm{k} \Omega$ 


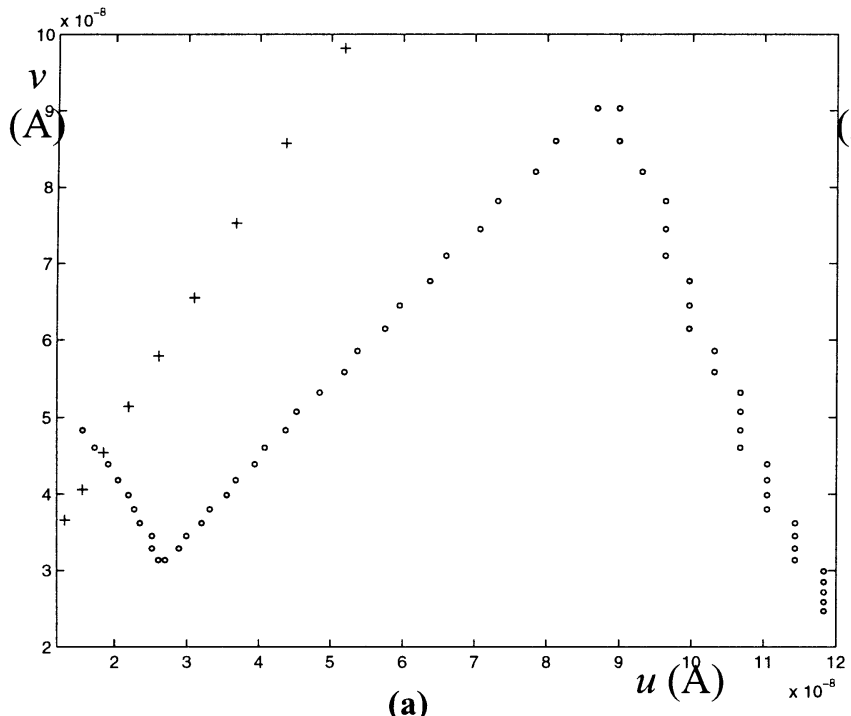

(a)

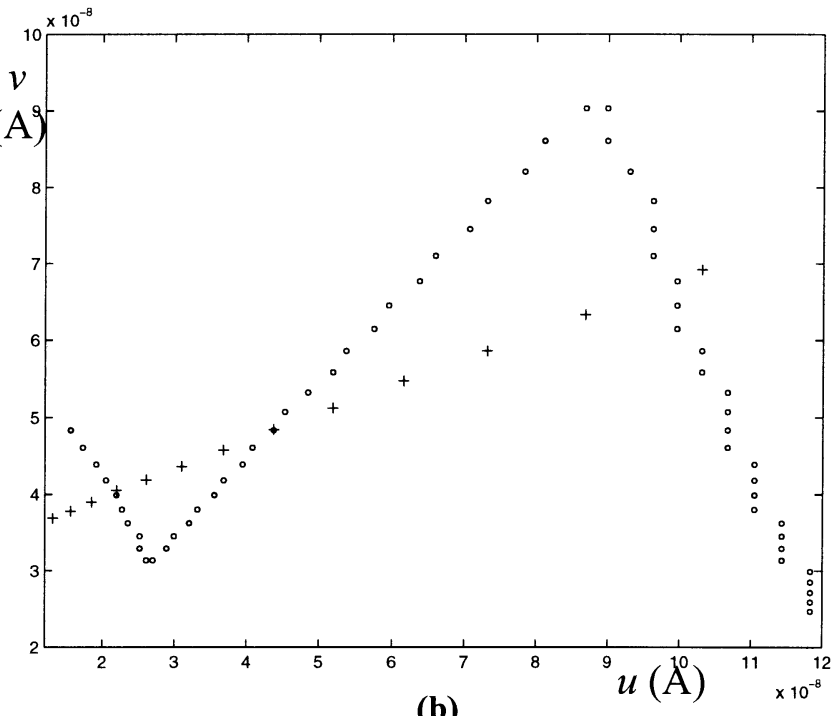

(b)



(c)

Fig. 18. Experimentally measured isoclines of (a) an excitable medium, (b) a biestable medium, and (c) an oscillatory medium.

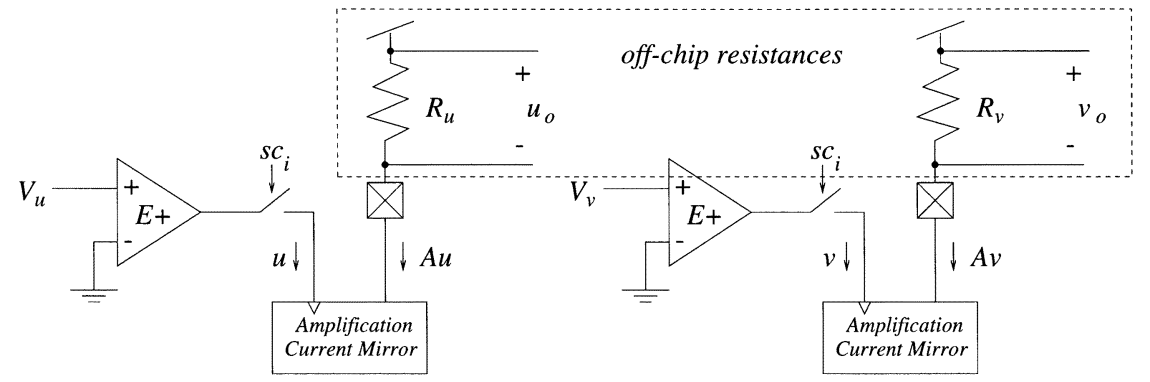

Fig. 19. Schematic of the additional circuitry to observe the cell state variables $u$ and $v$.

and $R_{v}=25 \mathrm{k} \Omega$. The observed state variables are the voltage drops in these resistances: $u_{o}$ and $v_{o}$ in Fig. 19.

We have experimentally measured transient results for three different settings of the cell parameters that reproduce three different spatio-temporal behaviors: a traveling wave, a trigger wave propagation and an oscillatory condition.

In our prototype, three different reference currents are used: $I_{\mathrm{REF} \_S}, I_{\mathrm{REF} \_\mathrm{E}}$, and $I_{\mathrm{REF} \_\mathrm{F}}$. The circuit parameters $I_{s}, I_{g}$, and
$I_{d u}$ are set through 5-bit DAC converters controlled by reference current $I_{\text {REF_S }}$. Each of these parameters can be varied in the range $\left[0,2 I_{\mathrm{REF} \_\mathrm{S}}\right]$ in steps of $I_{\mathrm{REF} \_\mathrm{S}} / 16$. The parameters $I_{e}$ and $I_{\gamma}$ are set through 5-bit DAC converters controlled by the second reference current $I_{\text {REF_E. Parameters }} I_{\beta}, I_{u o}, u_{1}$, and $u_{2}$ and the ones to set the initial conditions $u_{01}, u_{02}, v_{01}$, and $v_{02}$ are derived from 5-bit DAC converters controlled by reference current $I_{\text {REF_F }}$. The use of three reference currents allows an 

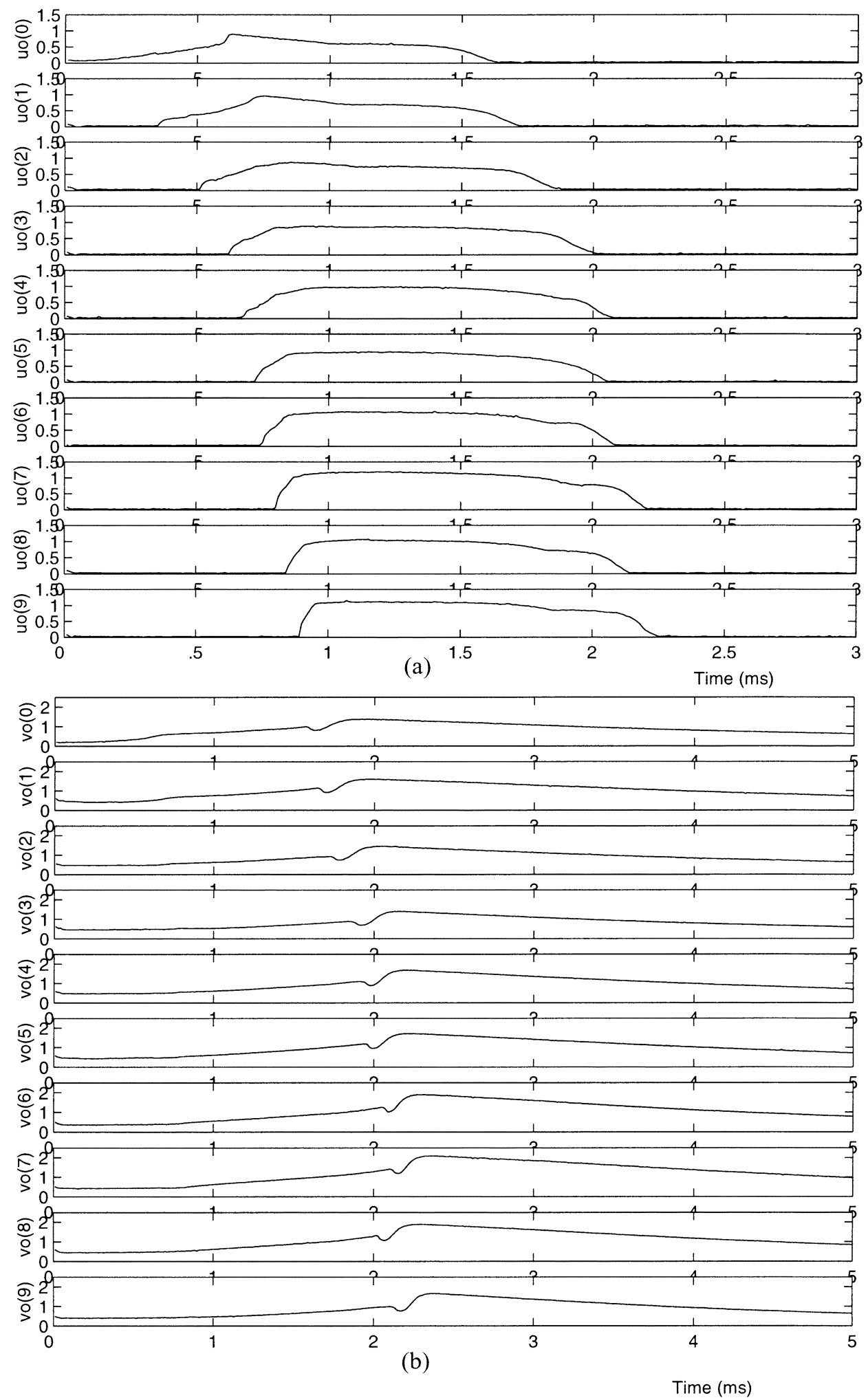

Fig. 20. Experimental results of a travelling wave propagating through a 1-D array of ten cells. (a) Waveforms of the $u$ state variables versus time. (b) Waveforms of the $v$ state variable versus time.

improved control of the system time constants and of the state variable values.

Fig. 20(a) and (b) shows experimental results of the travelling wave behavior. In the measurement of Fig. 20, the reference currents were set to $I_{\mathrm{REF} \_\mathrm{S}}=1 \mathrm{nA}$,
$I_{\mathrm{REF} \_\mathrm{E}}=1 \mathrm{nA}$ and $I_{\mathrm{REF} \_\mathrm{F}}=45 \mathrm{nA}$. The circuit parameters in these measurements were set to the following values: $I_{s}=I_{\mathrm{REF} \_\mathrm{S}}, I_{e}=(5 / 16) I_{\mathrm{REF} \_\mathrm{E}}, I_{\beta}=(1 / 16) I_{\mathrm{REF} \_\mathrm{F}}$, $I_{\gamma}=(3 / 16) I_{\mathrm{REF} \_\mathrm{E}}, I_{g}=I_{\mathrm{REF} \_\mathrm{S}}, I_{u o}=(0 / 16) I_{\mathrm{REF} \_\mathrm{F}}$, for the nonlinear block, $m_{10}=3.2, m_{20}=3.2, m_{0}=1$, 


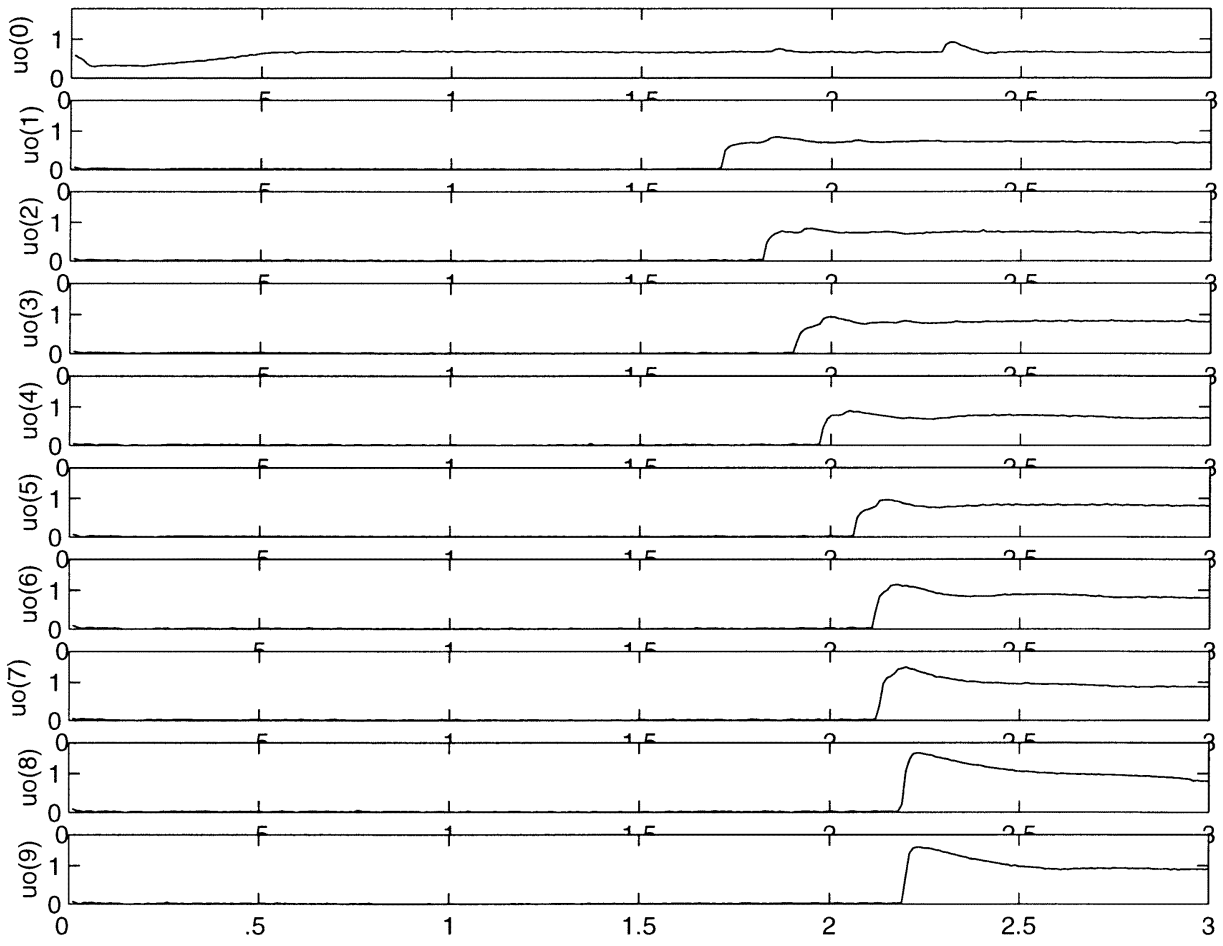

(a)

Time (ms)

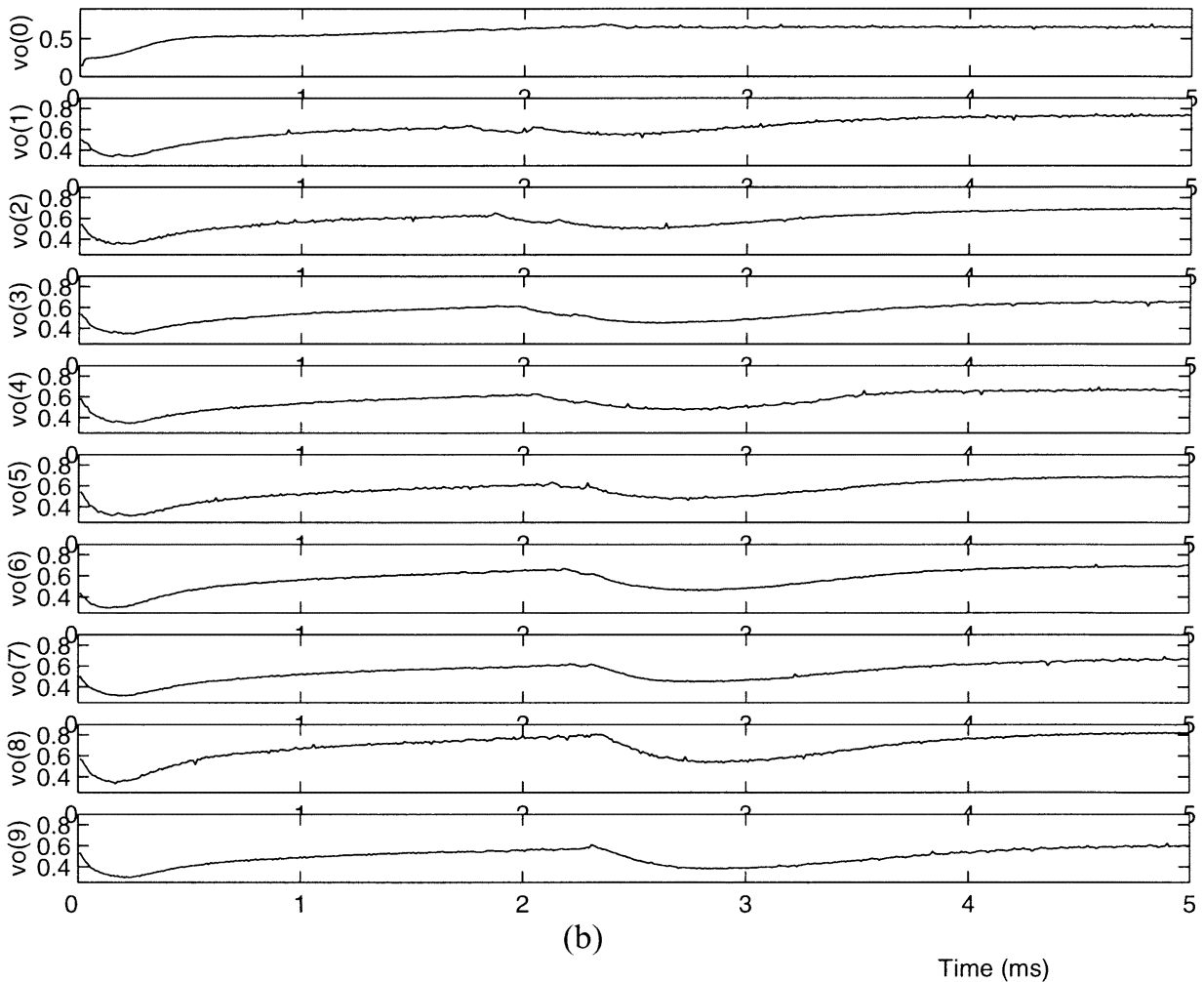

Fig. 21. Experimental measurements of a trigger wave propagating through a 1-D array of ten cells. (a) Waveforms of the $u$ state variables versus time. (b) Waveforms of the $v$ state variables versus.

$u_{1}=(10 / 16) I_{\mathrm{REF}-\mathrm{F}}, u_{2}=(28 / 16) I_{\mathrm{REF}-\mathrm{F}}$, and for the coupling elements $I_{d u}=(18 / 16) I_{\mathrm{REF} \_\mathrm{S}}$. Experimentally, we observed that each uncoupled cell has a unique equilibrium point (excitable medium) in which the state variables settle to the values $u_{0}=(1 / 16) I_{\mathrm{REF}-F}, v_{0}=(12 / 16) I_{\mathrm{REF}-F}$. All the cells in the array are initialized to their equilibrium state except for the first cell which is initialized to a nonresting state $u=(1 / 16) I_{\mathrm{REF}-F}, v=(1 / 16) I_{\mathrm{REF}-F}$. As can be observed in Fig. 20, this cell becomes excited going back to the equilibrium state after a refractory period. The perturbation of the first cell affects (through the coupling synapses) the next cell, which becomes also excited and leaves its resting state 

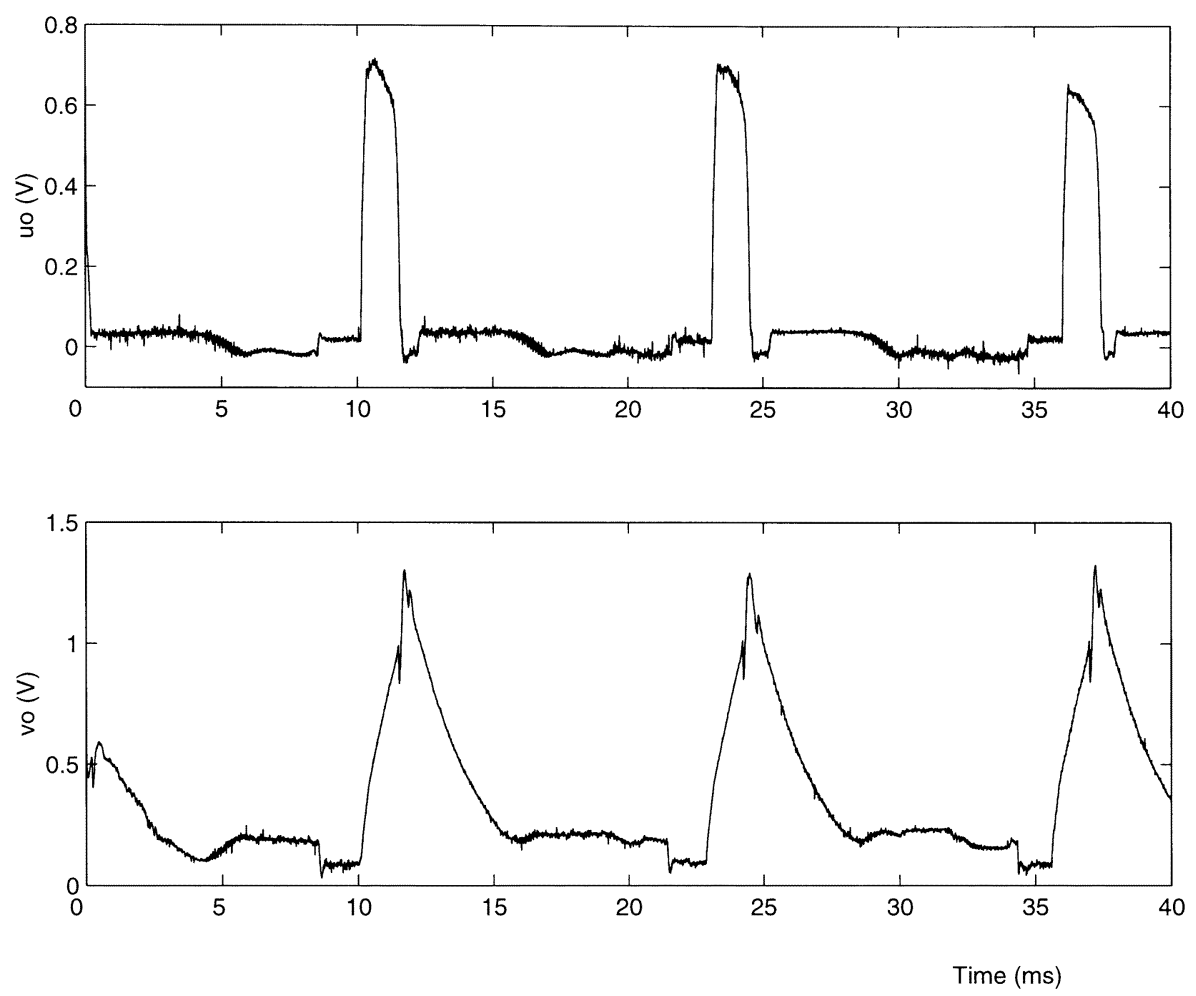

Fig. 22. Measured waveforms of the (a) $u$ and (b) $v$ state variables for an oscillatory medium.

during a refractory time, exciting also the third array cell. This way, a travelling wave propagates through the array. The total delay that occurs between the perturbation of the first cell (by the initial condition circuitry) and the perturbation affecting the tenth cell in the array is approximately $0.9 \mathrm{~ms}$. For these biasing conditions, the power consumption of the circuits generating the common biasing currents is $5.5 \mu \mathrm{W}$. The power consumption of each cell in the steady state is $3.5 \mu \mathrm{W}$ from a power supply voltage of $3.3 \mathrm{~V}$.

Fig. 21 shows results of a trigger wave propagating through the array. The reference currents were also set to $I_{\text {REF_S }}=$ $1 \mathrm{nA}, I_{\mathrm{REF}-\mathrm{E}}=1 \mathrm{nA}$ and $I_{\mathrm{REF}-\mathrm{F}}=45 \mathrm{nA}$. The circuit parameters were set to: $I_{s}=I_{\mathrm{REF}_{-} \mathrm{S}}, I_{e}=(3 / 16) I_{\mathrm{REF}-\mathrm{E}}$, $I_{\beta}=2 I_{\mathrm{REF} \_\mathrm{F}}, I_{\gamma}=2 I_{\mathrm{REF} \_\mathrm{E}}, I_{g}=I_{\mathrm{REF} \_\mathrm{S}}, I_{u o}=0$, for the nonlinear block, $m_{10}=3.2, m_{20}=3.2, m_{0}=1$, $u_{1}=(10 / 16) I_{\text {REF_F }}, u_{2}=2 I_{\text {REF_F }}$, and for the coupling elements $I_{d u}=(2 / 16) I_{\mathrm{REF} \_S}$. Fig. 21(a) shows the waveforms of the $u$ state variable versus time, while Fig. 21(b) depicts the waveforms of the $v$ state variable versus time. Experimentally, we observed that the uncoupled cell has two equilibrium points (biestable medium) in which the state variables settle to one of the pair of values: $\left(u_{01}=2 I_{\mathrm{REF}_{-} \mathrm{F}}, v_{01}=(14 / 16) I_{\mathrm{REF}_{-} \mathrm{F}}\right)$, $\left(u_{02}=(1 / 16) I_{\mathrm{REF}-F}, v_{02}=(12 / 16) I_{\mathrm{REF}-F}\right)$. All the cells in the array are initialized to the second equilibrium state $\left(u_{02}\right.$, $v_{02}$ ) except for the first one which is initialized to a nonresting condition $u=2 I_{\mathrm{REF} \text { F }}, v=0 \mathrm{nA}$ ). The first cell evolves toward the equilibrium state $\left(u_{01}, v_{01}\right)$ and perturbs (through the coupling synapses) the second cell which is triggered also to the $\left(u_{01}, v_{01}\right)$ equilibrium state. The perturbation is propagated through the array until all the cells have been triggered to the $\left(u_{01}, v_{01}\right)$ state. The time that occurs between the triggering of the second and the tenth cells in the array is approximately $0.5 \mathrm{~ms}$. For these biasing conditions, the power consumption of the circuits generating the common biasing currents is $6.5 \mu \mathrm{W}$. The power consumption of each cell in the $\left(u_{01}, v_{01}\right)$ equilibrium state is $5.6 \mu \mathrm{W}$. The power consumption is $3.9 \mu \mathrm{W}$ for each cell in the $\left(u_{02}, v_{02}\right)$ equilibrium state.

Fig. 22 shows the measured waveforms of the $u$ (upper trace) and $v$ (lower trace) state variables of one of the cells in the array when the circuit parameters are set to emulate an oscillatory medium. In this case, each isolated cell has only one unstable equilibrium point. Independently of the initial conditions, during its normal operation each cell describes a limit cycle around the equilibrium point. The reference currents were again

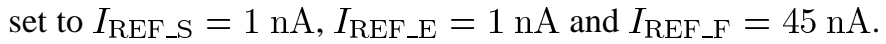
The circuit parameters used in this measurement were: $I_{s}=$ $I_{\mathrm{REF} \_\mathrm{S}}, I_{e}=(5 / 16) I_{\mathrm{REF} \_\mathrm{E}}, I_{\beta}=0 \mathrm{~A}, I_{\gamma}=(4 / 16) I_{\mathrm{REF} \_\mathrm{E}}$, $I_{g}=I_{\mathrm{REF} \_\mathrm{S}}, I_{u o}=0 \mathrm{~A}$, for the nonlinear block, $m_{10}=3.2$, $m_{20}=3.2, m_{0}=1, u_{1}=(10 / 16) I_{\mathrm{REF}-F}, u_{2}=2 I_{\mathrm{REF}-F}$, and for the coupling elements $I_{d u}=0 \mathrm{~A}$.

\section{CONCLUSION}

A second-order reaction-diffusion equation has been selected which is the simplest equation able to generate, through parameter setting, trigger wave, traveling wave propagation, spiral wave and Turing pattern formation.

A hardware implementing the spatially discretized version of the selected equation has been designed. In the hardware implementation the cell state variables are logarithmically encoded as voltages stored in some capacitors. This logarithmic compression of the state variables allows several decades of variation of 
these state variables without saturation of the elements and with low distortion. All the parameters of the implemented equation are set as biasing currents that can be adjusted several decades. Furthermore, by scaling some biasing currents of the circuit the time constant of the implemented equation can also be appropriately scaled. This can be helpful to make the waveforms generated on chip easily observable and for some applications where an extremely slow time constant may be needed (i.e., in locomotion applications).

A 1-D array of ten coupled cells has been integrated. The correct operation of the designed hardware has been verified through experimental measurements. We have performed a detailed characterization of the behavior of one isolated cell. Also, a traveling wave and a trigger wave propagating in the array have been experimentally measured.

\section{ACKNOWLEDGMENT}

The authors wish to acknowledge Prof. A. RodriguezVazquez and Prof. T. Roska for the financial support of the first steps of this work.

\section{REFERENCES}

[1] J. D. Murray, Mathematical Biology. New York: Springer-Verlag, 1989.

[2] V. N. Bitashev, A. V. Holden, and H. Zhang, "A model for the action of the external current onto excitable tissue," Int. J. Bifurcation Chaos, vol. 7, no. 2, 1997.

[3] V. N. Bitashev and A. V. Holden, "Re-entrant waves and their elimination in a model of mammalian ventricular tissue," Chaos, vol. 8, no. 1, 1998.

[4] V. I. Krinsky, V. N. Bitashev, and I. R. Efimov, "Autowaves principles for parallel image processing," Phys. D, vol. 49, pp. 247-253, 1991.

[5] M. Gómez-Gesteira, M. de Castro, V. Pérez-Villar, and L. O. Chua, "Experimental Chua's circuit arrays as an autowave simulator," IEEE Trans. Circuits Syst. I, vol. 46, Apr. 1999.

[6] P. Arena, M. Branciforte, G. Di Bernardo, M. Lavorgna, and L. Occhipinti, "Reaction-Diffusion CNN chip. Part I: IC implementation," presented at the Int. Symp. Circuits Systems, Geneva, Switzerland, 2000.

[7] R. Carmona, J. Jiménez-Garrido, R. Domínguez-Castro, S. Espejo, and A. Rodríguez-Vázquez, "Bio-Inspired analog parallel array processor chip with programmable spatio-temporal dynamics," presented at the Int. Symp. Circuits Systems, 2002.

[8] R. A. Fisher, "The wave of advance of advantageous genes," Ann. Eugenics, vol. 7, pp. 355-369, 1937.

[9] R. FitzHugh, "Mathematical models of excitation and propagation nerve," in Biol. Eng., H. Schwan, Ed. New York: McGraw-Hill, 1969.

[10] A. L. Hodgkin and A. F. Huxley, "A quantitative description of membrane current and its application to conduction and excitation in nerve," J. Physiol., vol. 117, pp. 500-544, 1952.

[11] J. J. Thyson, The Belousov-Zhabotinskii Reaction. New York: Springer-Verlag, 1976, vol. 10, Lecture Notes in Biomathematics.

[12] A. M. Turing, "The chemical basis of morphogenesis," Philos. Trans. $R$. Soc. London, ser. B, vol. 237, pp. 37-72, 1952

[13] R. J. Field and R. M. Noyes, "Oscillations in chemical systems, IV. Limit cycle behavior in a model of a real chemical reaction," J. Chem. Phys., vol. 60, pp. 1877-1884, Mar. 1974.

[14] A. Pérez-Muñuzuri, V. Pérez-Muñuzuri, V. Pérez-Villar, and L. O. Chua, "Spiral waves on a 2-D array of nonlinear circuits," IEEE Trans. Circuits Syst. I, vol. 40, Nov. 1993.
[15] V. Pérez-Muñuzuri, V. Pérez-Villar, and L. O. Chua, "Autowaves for image processing on a two-dimensional CNN array of excitable nonlinear circuits: Flat and wrinkled labyrinths," IEEE Trans. Circuits Syst. I, vol. 40, Mar. 1993.

[16] T. Serrano-Gotarredona and A. Rodríguez-Vázquez, "On the design of second order dynamics reaction-diffusion CNN's," J. VLSI Signal Processing, vol. 23, pp. 351-371, Nov. 1999.

[17] A. G. Andreou and K. A. Boahen, "Translinear circuits in subthreshold MOS," Analog Integrated Circuits and Signal Processing, vol. 9, pp. 141-166, 1996.

[18] B. Linares-Barranco, T. Serrano-Gotarredona, and R. Serrano-Gotarredona, "On compact low- power calibration mini-DAC's for neural massive arrays," IEEE Trans. Neural Networks, vol. 14, pp. -, Sept. 2003

[19] D. R. Frey, "State-Space synthesis and analysis of log-domain filters," IEEE Trans. Circuits Syst. II, vol. 45, pp. 1205-1211, Sept. 1998.

[20] - "Log-Domain filtering: An approach to current mode filtering," in Proc. Inst. Elect. Eng., vol. 140, Dec. 1996, pp. 406-416.

[21] — - "Exponential state space filters: A generic current mode design strategy," IEEE Trans. Circuits Syst. I, vol. 43, pp. 34-42, Jan. 1996.

[22] "Special section on instantaneous companding in analog signal processing," IEEE Trans. Circuits Syst. II, vol. 45, Sept. 1998.

[23] E. Vittoz and J. Fellrath, "CMOS analog integrated circuits based on weak inversion operation," IEEE J. Solid State Circuits, vol. 12, pp. 224-231, June 1997.

[24] B. Gilbert, "Translinear circuits: A proposed classification," Electron. Lett., vol. 11, no. 1, pp. 14-16, 1975.

[25] B. L. Hart, "Translinear circuit principle: A Reformulation," Electron. Lett., vol. 15, no. 24, pp. 801-803, Nov. 1979.

[26] T. Serrano-Gotarredona and B. Linares-Barranco, "Current mode fully programmable piece wise linear block for neuro fuzzy applications," Electron. Lett., vol. 38, no. 20, pp. 1165-1166, Sept. 2002.

[27] G. Cauwenberghs and A. Yariv, "Fault-Tolerant dynamic multi-level storage in analog VLSI," IEEE Trans. Circuits Syst. II, vol. 41, pp. 827-829, Dec. 1994.

[28] L. Pivka, "Autowaves and spatio-temporal chaos in CNNs- Part I: A tutorial," IEEE Trans. Circuit s Syst. I, vol. 42, Oct. 1995.

[29] T. Serrano-Gotarredona, B. Linares-Barranco, and A. G. Andreou, "Very wide range tunable CMOS/bipolar current mirrors with voltage clamped input," IEEE Trans. Circuits Syst. II, Nov. 1999.

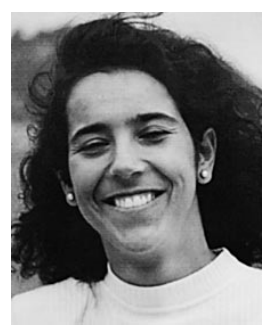

Teresa Serrano-Gotarredona (S'95-A'00) received the B.S. degree in electronic physics in June 1992 and the Ph.D degree in VLSI neural categorizers in December 1996 from the University of Seville, Sevilla, Spain, after completing all her research at the Sevilla Microelectronics Institute (IMSE), which is one of the institutes of the National Microelectronics Center (CNM) of the Spanish Research Council (CSIC). She received the M.S. degree from the Department of Electrical and Computer Engineering, Johns Hopkins University, Baltimore, MD.

She was on a sabbatical stay at the Electrical Engineering Department, Texas A\&M University, College Station, during spring 2002. From 1998 to 2000, she was Assistant Professor at the University of Seville. Since June 2000, she has held a Tenured Scientist position at the Sevilla Microelectronics Institute (IMSE), Sevilla, Spain. Her research interests include analog circuit design of linear and nonlinear circuits, very large-scale integration (VLSI) neural-based pattern recognition systems, VLSI implementations of neural computing and sensory systems, transistor parameters mismatch characterization, address-event-representation VLSI, $R F$ circuit design, and real-time vision processing chips.

Dr. Serrano-Gotarredona was corecipient of the 1997 IEEE TRANSACTIONS ON VLSI SYSTEMS Best Paper Award for the paper "A Real-Time Clustering Microchip Neural Engine" and of the IEEE CAS Darlington Award for the paper "A General Translinear Principle for Subthreshold MOS Transistors." She is Coauthor of the book "Adaptive Resonance Theory Microchips." She was sponsored by a Fulbright Fellowship during her time at Johns Hopkins. 


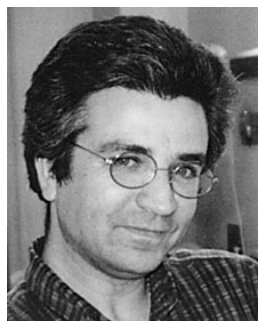

Bernabe Linares-Barranco (S'90-M'92) received the B.S. degree in electronic physics in June 1986 and the M.S. degree in microelectronics in September 1987, both from the University of Seville, Sevilla, Spain. He received the Ph.D. degree in high-frequency OTA-C oscillator design in June 1990 from the University of Seville, Spain, and the $\mathrm{Ph} . \mathrm{D}$, degree in analog neural-network design in December 1991 from Texas A\&M University, College Station.

Since September 1991, he has been a Tenured Scientist at the Sevilla Microelectronics Institute (IMSE), which is one of the institutes of the National Microelectronics Center (CNM) of the Spanish Research Council (CSIC). In January 2003, he was promoted to Tenured Researcher. From September 1996 to August 1997, he was on sabbatical stay at the Department of Electrical and Computer Engineering of the Johns Hopkins University, Baltimore, as a Postdoctoral Fellow. During spring 2002, he was Visiting Associate Professor at the Electrical Engineering Department, Texas A\&M University. He has been involved with circuit design for telecommunication circuits, VLSI emulators of biological neurons, very large-scale integration (VLSI) neural-based pattern recognition systems, hearing aids, precision circuit design for instrumentation equipment, bio-inspired VLSI vision processing systems, transistor parameters mismatch characterization, address-event-representation VLSI, RF circuit design, and real-time vision processing chips. He is coauthor of the book "Adaptive Resonance Theory Microchips."

Dr. Linares-Barranco was corecipient of the 1997 IEEE TRANSACTIONS ON VLSI SYSTEMS Best Paper Award for the paper "A Real-Time Clustering Microchip Neural Engine," and of the 2000 IEEE CAS Darlington Award for the paper "A General Translinear Principle for Subthreshold MOS Transistors." He organized the 1994 Nips Post-Conference Workshop "Neural Hardware Engineering." From July 1997 to July 1999, he was Associate Editor of the IEEE TRANSACTIONS ON CIRCUITS AND SYSTEMS PART II, and since January 1998, he has been Associate Editor for the IEEE TRANSACTIONS ON NEURAL NETWORKS. He is Chief Guest Editor of the 2003 IEEE TRANSACTIONS ON NEURAL NETWORKS Special Issue on Neural Hardware Implementations. 